\title{
Ginsenoside Rg5 increases cardiomyocyte resistance to ischemic injury through regulation of mitochondrial hexokinase-II and dynamin-related protein 1
}

\author{
Yi-Lin Yang ${ }^{1}$, Jia Li ${ }^{1}$, Kang Liu ${ }^{1,2}$, Lei Zhang ${ }^{1}$, Qun Liu ${ }^{1}$, Baolin Liu ${ }^{1,2}$ and Lian-Wen Qi ${ }^{\star, 1}$
}

\begin{abstract}
Hexokinase-II (HK-II) and dynamin-related protein 1 (Drp1) regulate mitochondrial function differently. This study was designed to investigate the cardioprotective effect of ginsenoside Rg5 (Rg5) with emphasis on the regulation of mitochondrial HK-II and Drp1. Saturated acid palmitate $(\mathrm{PA})$ stimulation increased lactate accumulation and induced cellular acidification by impairing the activity of pyruvate dehydrogenase (PDH) in cardiomyocytes, leading to HK-II dissociation from mitochondria. Rg5 improved PDH activity and prevented cellular acidification by combating fatty-acid oxidation, contributing to protecting mitochondrial HK-II. HK-II binding to mitochondria prevented mitochondrial Drp1 recruitment, whereas Drp1 activation decreased the content of mitochondrial HK-II, demonstrating the reciprocal control for binding to mitochondria. Rg5 promoted Akt translocation to mitochondria and increased HK-II binding to mitochondria while coordinately suppressing Drp1 recruitment and mitochondrial fission. Akt inhibitor triciribine or knockdown of Akt with small interfering RNA diminished the effects of Rg5, indicating that Rg5 inhibited Drp1 activation and promoted HK-II mitochondrial binding through Akt activation. Rg5 prevented the opening of mitochondrial permeability transition pore and increased ATP production, resultantly increasing cardiomyocyte resistance to hypoxia/reoxygenation injury. Meanwhile, Rg5 prevented cell apoptosis with increased HK-II binding and reduced Drp1 recruitment to mitochondria in isoproterenol-induced ischemic heart of mice. Taken together, these findings not only established a previously unrecognized role of ginsenosides in cardioprotection but also suggest that mitochondrial HK-II binding and Drp1 recruitment could be targeted therapeutically to prevent ischemic injury in the heart.
\end{abstract}

Cell Death and Disease (2017) 8, e2625; doi:10.1038/cddis.2017.43; published online 23 February 2017

The heart has a very high energy demand and uses fatty acid and glucose as energy substrates. Although the heart prefers fatty-acid oxidation for energy production, glucose is an efficient energy supply because the amount of ATP produced per $\mathrm{O}_{2}$ consumed is greater in glucose oxidation compared with fatty-acid oxidation. ${ }^{1}$ Fatty-acid oxidation and glucose oxidation compete for substrates, and the reciprocal control between fatty-acid and glucose metabolism is described as the Randle Cycle. ${ }^{2}$ Increased myocardial fatty-acid oxidation is often observed in patients with diabetes mellitus and obesity owing to the elevated circulating fatty acids and insulin resistance $^{3-5}$ In mitochondria, enhanced fatty-acid oxidation decreases glucose oxidation by inhibiting the activity of pyruvate dehydrogenase $(\mathrm{PDH})$, the rate-limiting enzyme linking glycolysis to glucose oxidation, and then impairs cardiac energy efficiency, especially during ischemia/reperfusion (I/R). ${ }^{6,7}$ The alternation of mitochondrial oxidation in the heart is considered the main cause for the vulnerability to ischemic injury in metabolic disoreders. ${ }^{7}$

Hexokinase $(\mathrm{HK})$ catalyzes the first step in glycolysis by converting glucose to glucose 6-phosphate, and HK-II is the predominant isoform in the heart. HK-II enhances aerobic glycolysis in tumor cells (the Warburg effect) and thereby provides tumor cells with resilience to cell death. ${ }^{8}$ In cardiomyocytes, HK-II dynamically shuttles between the mitochondria and cytoplasm in response to cellular stress. Hence dysregualtion of mitochondrial HK-II has a critical influence on the susceptibility of the heart to I/R injury. ${ }^{9} \mathrm{HK}-\mathrm{II}$ binds to outer mitochondrial membrane via connection with voltage-dependent anion channel 1 (VDAC), which interacts with the adenine nucleotide translocase (ANT), forming a contact site between the outer and inner membranes. ${ }^{10}$ This complex is essential for the permeability barrier of the inner mitochondrial membrane (IMM) and the mitochondrial permeability transition pore (mPTP) acts as a key nodal point in mediating mitochondrial function. Historically, it was suggested that the MPTP was comprised of VDAC in the outer mitochondrial membrane and ANT in the IMM. In the past decade, however, genetic studies demonstrate that ANT and $F_{1} F_{0}$ ATP synthase are the core constituents of the complex, responsible for mitochondrial homeostasis and energy generation. ${ }^{11}$ Moreover, a recent study demonstrates the implication of cardiac cAMP signaling in mitochondrial permeability transition. ${ }^{12}$ Loss of the permeability barrier of IMM is considered the final event causing cell death during $\mathrm{I} / \mathrm{R}$ injury. ${ }^{13}$ HK-Il detachment from mitochondria induces a conformational change of the molecular complex, leading to mitochondrial depolarization and cell death. ${ }^{9,14}$ Therefore,

\footnotetext{
${ }^{1}$ State Key Laboratory of Natural Medicines, China Pharmaceutical University, Nanjing, China and ${ }^{2}$ Jiangsu Key Laboratory of TCM Evaluation and Translational Research, Department of Pharmacology of Chinese Materia Medica, China Pharmaceutical University, Nanjing, China

${ }^{*}$ Corresponding author: L-W Qi, State Key Laboratory of Natural Medicines, China Pharmaceutical University, Tongjia Lane, \#639 Longmian Avenue, Jiangning District, Nanjing 211198, China. Tel: +86 25 86185559; Fax: +86 25 83271379; E-mail: Qilw@cpu.edu.cn

Received 01.9.16; revised 13.12.16; accepted 18.1.17; Edited by M Campanella
} 
mitochondrial HK-II dissociation during ischemia is correlated with cytochrome $\mathrm{C}$ release, ROS production and infarct size. ${ }^{14}$

Mitochondrial HK-II orchestrates metabolic and anti-cell death effects, contributing to mitochondrial homeostasis. Maintenance of functional and structural integrity of mitochondria is crucial for HK-II binding. Mitochondria are dynamic organelles and their morphological integrity is maintained by a delicate balance between mitochondrial fusion and fission. In response to ischemic stress, mitochondria undergo fragmentation, a fission process that is dependent on the activation of mitochondrial fission protein dynamin-related protein 1 (Drp1), resulting in mitochondrial dysfunction and apoptosis. ${ }^{15}$ In contrast, inhibiting mitochondrial fission prevents mPTP opening and reduces cell death. ${ }^{9}$ In the brain, mitochondrial fission occurs in ischemic stroke and precedes neuronal cell death. ${ }^{16}$ In addition, Drp1 activation and mitochondrial fission are demonstrated to be associated with endoplasmic reticulum stress, oxidative stress and mitochondrial dysfunction, which are mediators of $\mathrm{I} / \mathrm{R}$ injury. ${ }^{17-19}$ Though the relation between Drp1 activation and mitochondrial HK-II binding is not known, we hypothesized that maintenance of the structural integrity of mitochondria should protect cardiomyocytes against I/R injury by increasing mitochondrial HK-II binding. In tumor cells, HK-II induction is mediated through Akt/ mTORC1 pathway. $^{20}$ In cardiomyocytes, HK-II has an Akt consensus sequence at position Thr473, which can be directly phosphorylated by Akt activation. ${ }^{21,22}$ Mitochondria fission is mediated through Drp1 activation and inactivation of Drp1 by phosphorylating at Ser 637 residue is shown to prevent mitochondrial fission with its resultant consequences. ${ }^{18,19}$ Because both HK-II and Drp1 exert their actions in mitochondria with opposite effects on cardiomyocyte survival, it is

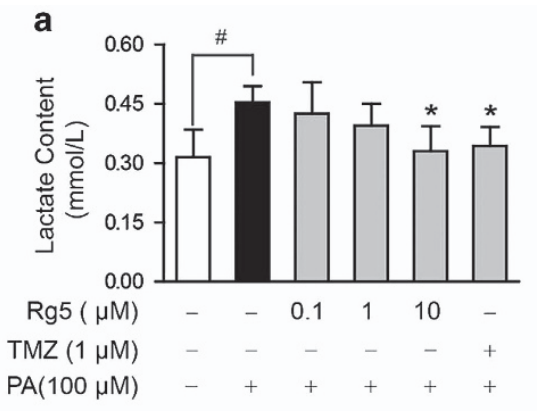

C

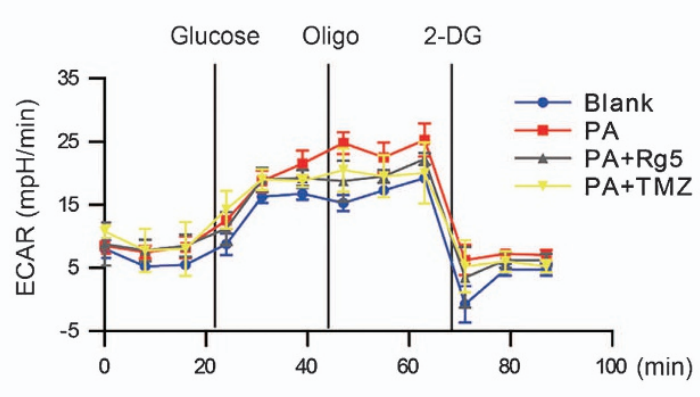

e

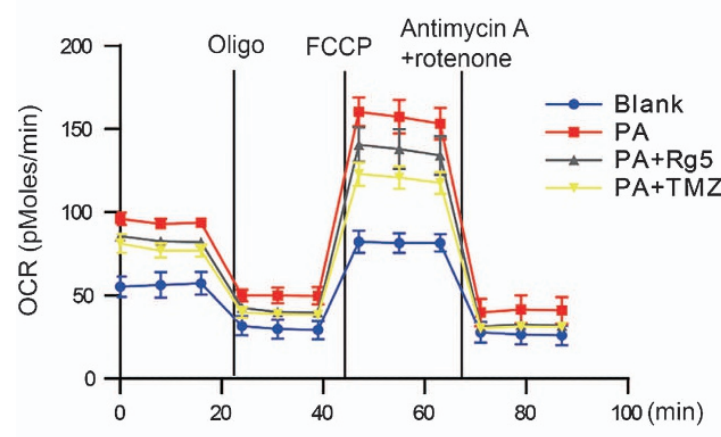

b

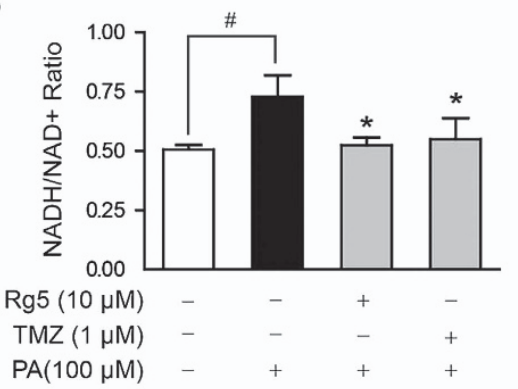

d
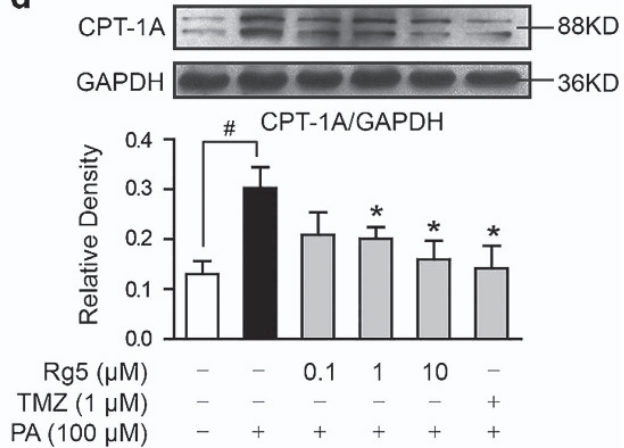

f

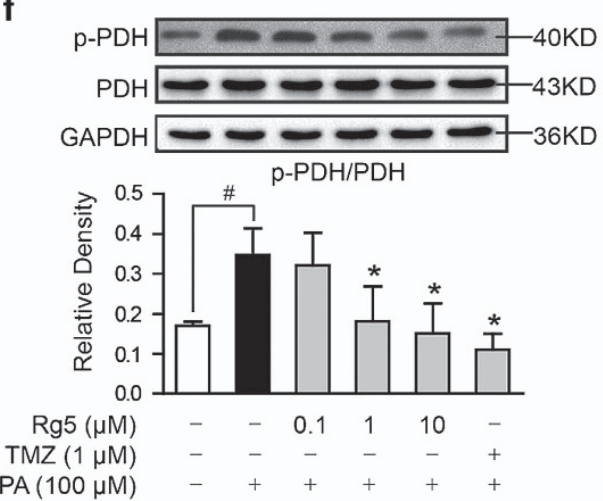

Figure $1 \mathrm{Rg} 5$ prevented acidification in cardiomyocytes. Neonatal rat ventricular myocytes (NRVMs) were stimulated with palmitate (PA, $100 \mu \mathrm{M})$ for $2 \mathrm{~h}$. (a and b) Lactate contents $(n=5)$ and NADH/NAD+ ratio $(n=5)$. (c) Extracellular acidification ratio (ECAR) in NRVMs was measured using an XFe96 Extracellular Flux Analyzer ( $n=4)$. (d) CPT-1 protein expression in NRVMs was determined with western blot $(n=3)$. (e) Oxygen consumption ratio (OCR) was measured using an XFe96 Extracellular Flux Analyzer $(n=4)$. (f) Phosphorylation of PDH was detected with western blot $(n=3)$. Data are expressed as mean \pm S.D. $P<0.05$ versus PA treatment; ${ }^{\#} P<0.05$ versus indicated treatment 
a

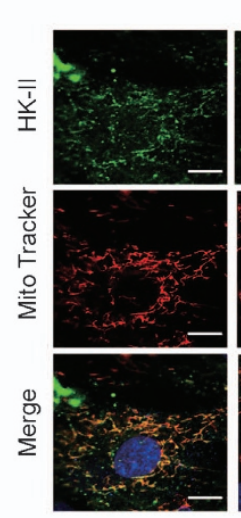

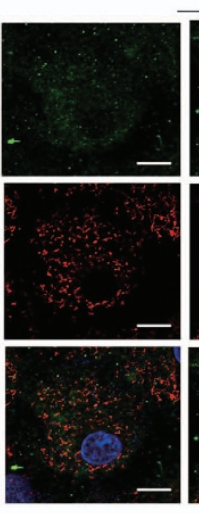

$-$

$-$

$\operatorname{Rg}(1 \mu \mathrm{M})$

Mdivi-1 $(10 \mu \mathrm{M})$

$\mathrm{PA}(100 \mu \mathrm{M})$

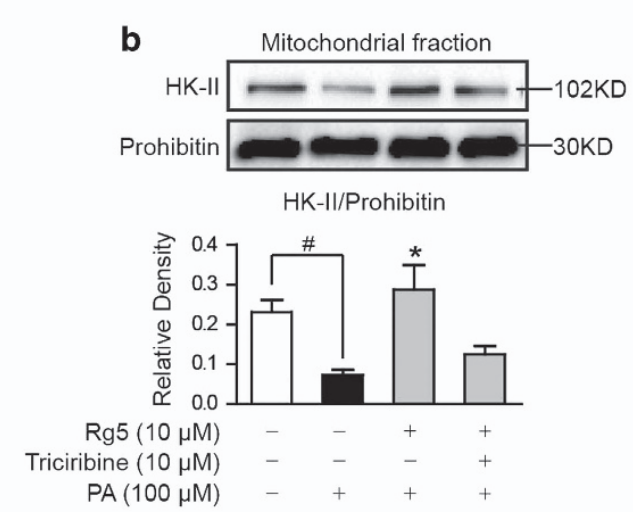

Mitochondrial HK-II Binding

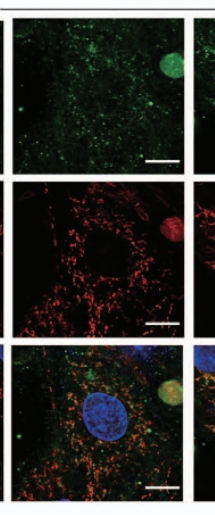

0.1

-

$+$

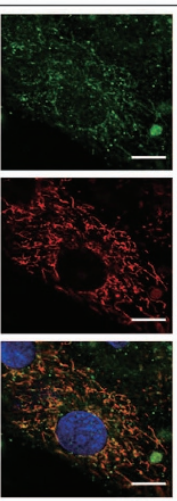

1

-
-
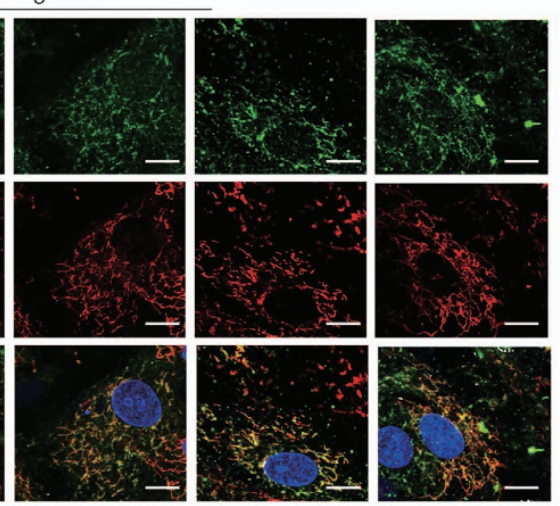

10
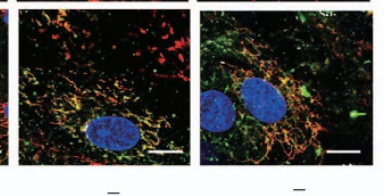

-
+

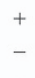

$-$

$-$

$+$

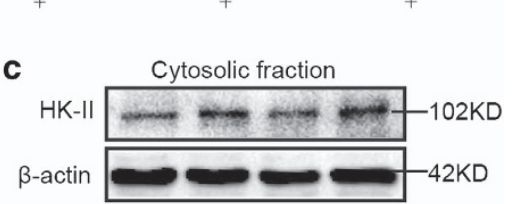

HK-II/ $\beta$-actin

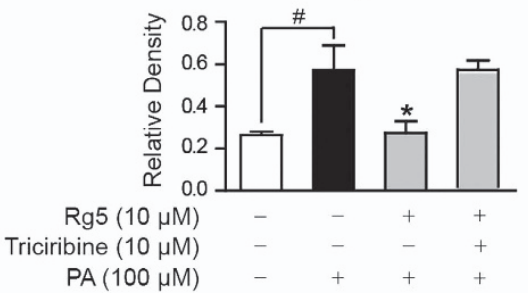

Figure 2 Rg5 prevented HK-II detachment from mitochondria. Neonatal rat ventricular myocytes (NRVMs) were stimulated with palmitate (PA, $100 \mu \mathrm{M})$ for $2 \mathrm{~h}$. (a) View of mitochondrial localization of HK-II with confocal scanning microscope (Green: HK-II; Red: MitoTracker Red cMXRos; Scale bars: $10 \mu \mathrm{m}$ ); (b and c) Mitochondrial and cytosolic HK-II expressions were determined by western blot. Data are expressed as mean \pm S.D. from three or four independent experiments. ${ }^{*} P<0.05$ versus $P A$ treatment; ${ }^{\#} P<0.05$ versus indicated treatment

tempting to know whether HK-II and Drp1 regulate each other and compete for binding to mitochondria, especially during I/R injury.

Panax ginseng is a traditional herbal medicine used for enhancing human health and ginsenosides are the major bioactive constituents in ginseng root. Ginsenosides have been reported to promote glucose uptake in insulin-sensitive tissues, prevent ectopic lipid deposition ${ }^{23-25}$ and demonstrated potentially positive effects in heart diseases. ${ }^{26}$ Ginsenoside Rg5 (Rg5) is the most abundant compound in steamed ginseng. ${ }^{27}$ This study was designed to investigate if Rg5 promotes resistance to I/R injury in cardiomyocytes via regulation of energy metabolism and mitochondrial function. In the present study, we found that Drp1 activation impaired HK-II binding to mitochondria, setting cardiomyocytes at a stage susceptible to I/R injury. Rg5 inhibited Drp1 activation and preserved mitochondrial HK-II via Akt activation, thereby protecting the cell against $\mathrm{I} / \mathrm{R}$ insult. These findings established a previously unrecognized role of ginsenosides in the regulation of mitochondrial function and suggest that mitochondrial Drp1 recruitment and HK-II binding could be targeted therapeutically to prevent I/R injury in the heart.

\section{Results}

Rg5 prevented acidification in cardiomyocytes. Palmitate (PA) stimulation increased lactate accumulation and NADH/ $\mathrm{NAD}^{+}$ratio in neonatal rat ventricular myocytes (NRVMs), reflecting an increase in the cytosolic redox state (Figures 1a and b). Rg5 treatment limited lactate production, reduced $\mathrm{NADH} / \mathrm{NAD}^{+}$ratio (Figures $1 \mathrm{a}$ and $\mathrm{b}$ ), and thereby prevented cellular acidification (Figure 1c). Carnitine palmitoyltransferase I (CPT-1) is a mitochondrial enzyme allowing fatty acids entry into mitochondria for oxidation. Rg5 prevented fatty-acid entry into mitochondria by downregulation of CPT-1 expression (Figure 1d) and reduced oxygen consumption ratio (OCR) (Figure 1e) in NRVMs, demonstrating its inhibitory effect on fatty-acid oxidation in mitochondria. $\mathrm{PDH}$ links glycolysis to glucose oxidation by converting pyruvate to acetyl-CoA for tricarboxylic acid cycle and thus shifts pyruvate from lactate to oxidation. Rg5 preserved PDH activity by dephosphorylation (Figure 1f), and this action should contribute to reducing lactate accumulation. Together, these results demonstrated that $\mathrm{Rg} 5$ reduced fatty-acid oxidation in mitochondria and prevented cellular acidification 
by reestablishing coupling of glycolysis to glucose oxidation. Trimetazidine, an anti-ischemic metabolic agent known as fatty-acid oxidation inhibitor, demonstrated a similar regulation as Rg5 (Figure 1).

Rg5 prevented HK-II detachment from mitochondria. Mitochondrial HK-II is susceptible to cellular acidification. ${ }^{14}$ Cardiac acidification induced HK-II to dissociate from mitochondria, but this alternation was prevented by Rg5 as well as trimetazidine treatment (Figure 2a). Mitochondrial fission inhibitor Midiv1 reduced HK-II detachment from mitochondria, suggesting the potential role of mitochondrial morphology in HK-II binding. Western blot showed that Rg5 restored HK-II expression in mitochondria and concomitantly reduced HK-II expression in the cytosol, further confirming that Rg5 prevented HK-II detachment from mitochondria in response to PA stimulation (Figures $2 b$ and $c$ ). Akt inhibitor triciribine diminished the effect of Rg5 on the protection of mitochondrial HK-II, suggesting Rg5 increased HK-II association with mitochondria via regulation of Akt (Figures $2 b$ and $c$ ).

Rg5 promoted Akt association with HK-Il in mitochondria. To know if Rg5 regulated mitochondrial HK-Il binding through Akt activation, we first performed molecular docking of Rg5 to Akt using the Autodock program. According to our docking result, Rg5 could bind to Akt by forming two stable hydrogen bonds with Glu 315 and Leu 317, as shown in Figure 3a. The binding of Rg5 probably induced the conformational changes of Akt, which would facilitate the phosphorylation at Ser 473. To validate the mimic analysis, we observed the effect of Rg5 in NRVMs, and found that Rg5 enhanced Akt phosphorylation (Ser 473), and significant effects were observed at 2 and $4 \mathrm{~h}$ (Figure $3 \mathrm{~b}$ ). Immunoprecipitation examination showed that phosphorylated Akt consensus sequence (PAS) presented in HK-II and Rg5 treatment increased PAS expression when cells were exposed to PA (Figure 3c). When HK-II was immunoprecipitated and then blotted using Akt or p-Akt (Ser 473) antibodies, Rg5 treatment increased total Akt and phosphorylated Akt expression in HK-Il protein in cardiomyocyte (Figure $3 \mathrm{~d}$ ), indicating that Rg5 promoted Akt binding to HK-II. Consistently, confocal scanning further confirmed that $\mathrm{Rg} 5$ promoted Akt to translocate to mitochondria and join HK$\mathrm{II}$, indicated by the increased blue staining in the merged image (Figure 3e). When PA stimulation led to HK-II and Akt detachment from mitochondria, the alternation was prevented by $\mathrm{Rg} 5$ treatment (Figure $3 \mathrm{e}$ ). We transfected $\mathrm{H} 9 \mathrm{c} 2$ cells with small interfering RNA (siRNA) to silence Akt and found that the effect of Rg5 on the increased mitochondrial HK-II was diminished (Figure 3f), further confirming that Rg5 promoted HK-II binding to mitochondria though regulation of Akt activation.

Rg5 inhibited Drp1 activation in cardiomyocytes. We then investigated the effect of Rg5 on Drp1 activation in NRVMs. The view of confocal microscopy showed that upon PA stimulation, Drp1 recruited to mitochondria and caused mitochondrial fragmentation in NRVMs, whereas Rg5 treatment inhibited Drp1 recruitment and normalized mitochondrial structure (Figures $4 \mathrm{a}$ and b), indicating that Rg5 prevented mitochondrial fission by inhibition of Drp1 activation. Trimezivi-1 also inhibited Drp1 activation and prevent mitochondrial fission (Figures $4 a$ and b). Phosphorylation of Drp1 at Ser 637 residue is proposed to inactivate Drp1. ${ }^{16}$ In NRVMs, Rg5 enhanced phosphorylation of Drp1 (Ser 637) and this regulation was preserved when cells were exposed to PA challenge, exerting the ability to suppress Drp1 activation (Figures 4c and d). Mito-TEMPO, a mitochondriatargeted antioxidant, also preserved Drp1 phosphorylation (Ser 637) in PA-stimulated NRVMs (Figure 4d). Akt inhibitor triciribine blocked Rg5 action in the regulation of Drp1 phosphorylation, indicative of the involvement of Akt activation in Rg5 action.

Rg5 inhibited Drp1 activation via regulation of Akt. We prepared Drp1 protein using immunoprecipitation method from NRVMs and found that PAS presented in Drp1 protein (Figure 5a), suggesting the regulatory potential of Akt. Similar to the regulation of HK-II, Rg5 treatment increased PAS and Akt expression in Drp1 (Figures 5a and b), indicating that Rg5 promoted Akt location on Drp1. In H9c2 cells, siRNA knockdown of Akt did not influence mitochondrial structure, but diminished the inhibitory effect of Rg5 on mitochondrial fission in PA-stimulated cells, indicating that Rg5 protected mitochondrial morphological integrity dependent on Akt activation (Figure $5 \mathrm{c}$ ).

To explore the potential interaction between Drp1 activation and mitochondrial HK-II, we observed mitochondrial HK-II in H9c2 cells when Drp1 was silenced by siRNA. PA stimulation reduced the content of $\mathrm{HK}-\mathrm{II}$ in mitochondria and this change was blocked by knockdown of Drp1, indicating that inhibition of Drp1 activation could increase HK-II binding to mitochondria (Figure 5d). 2-deoxy-D-glucose (2-DG) and insulin are proposed to reduce or increase HK-II binding to mitochondria, respectively. To know if mitochondrial HK-II influenced Drp1 activation, we observed Drp1 recruitment in the presence of 2DG or insulin, respectively. We found that treatment with 2-DG in NRVMs increased Drp1 recruitment to mitochondria, suggesting that the loss of mitochondrial HK-II promoted Drp1 recruitment to mitochondria (Figure 5e). In contrast, insulin prevented Drp1 recruitment to mitochondria in response to PA challenge (Figure $5 f$ ). Together, these results elucidated the reciprocal control between HK-II and Drp1 for the completion of mitochondrial binding.

Rg5 ameliorated mitochondrial dysfunction in cardiomyocytes. The direct consequence of mitochondrial HK-II disruption is mitochondrial dysfunction. In PA-stimulated NRVMs, mitochondrial ROS production (Figure 6a) and cellular calcium accumulation (Figure 6b) increased, indicative of oxidative stress and calcium overload (Figures $6 \mathrm{a}$ and b). These alternations were prevented by Rg5 treatment (Figures $6 a$ and b). As a result of improved mitochondrial homeostasis, Rg5 prevented the opening of mPTP (Figure $6 \mathrm{c}$ ) and restored the loss of the mitochondrial membrane potential $(\Delta \psi m)$ (Figure $6 \mathrm{~d}$ ), demonstrating the protective effect on mitochondrial function. Trimetazidine and Mdivi-1 demonstrated a similar regulation as $\mathrm{Rg} 5$. As a calcineurin inhibitor, cyclosporine A prevented mPTP opening in NRVMs (Figure 6c). In contrast, calcium ionophore 
ionomycin induced mPTP opening (Figure 6c). The different regulations suggested the involvement of calcium overload in PA-induced mPTP opening.
Rg5 increased cardiomyocyte resistance to I/R injury. Rg5 inhibited Drp1 activation and promoted HK-Il binding to mitochondria. Therefore, it was tempting to know whether

a

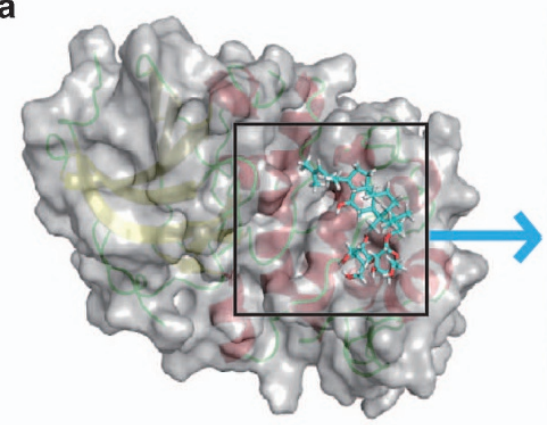

c

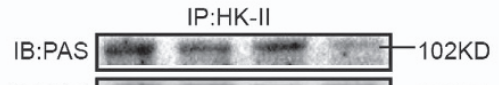

IB:HK-II -102KD

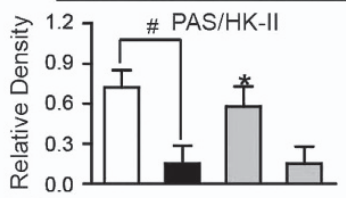

$\operatorname{Rg} 5(10 \mu \mathrm{M})$

Triciribine $(10 \mu \mathrm{M})$

$\mathrm{PA}(100 \mu \mathrm{M})$

e

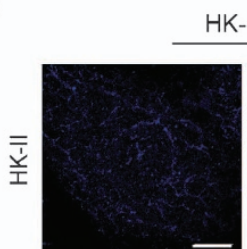

HK-II and p-Akt Co-localization in Mitochondrial

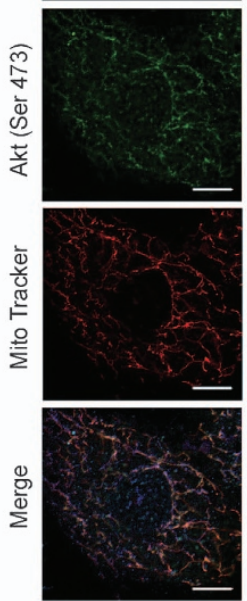

Blank
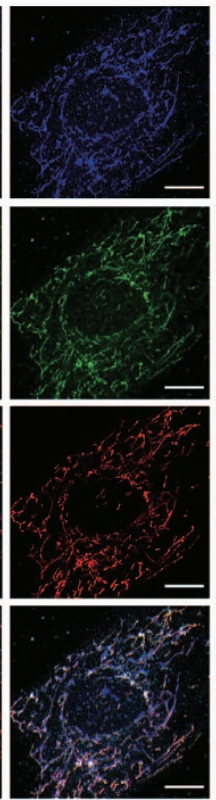

$10 \mu \mathrm{M}$ Rg5
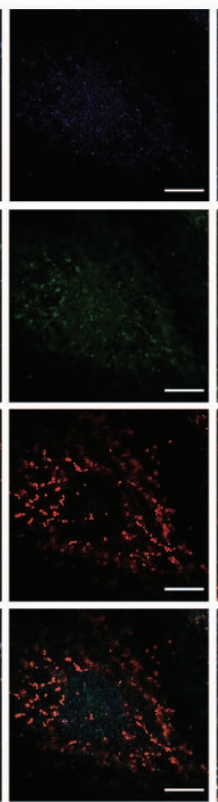

PA
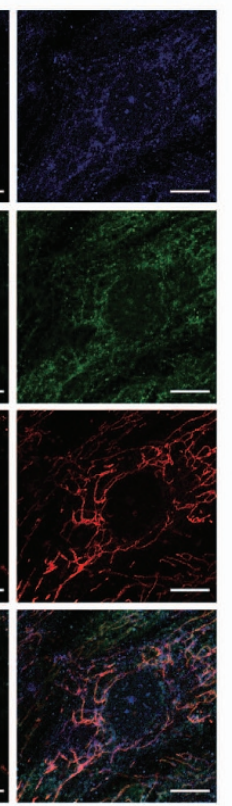

$\mathrm{PA}+$

$10 \mu \mathrm{M} \operatorname{Rg} 5$ b

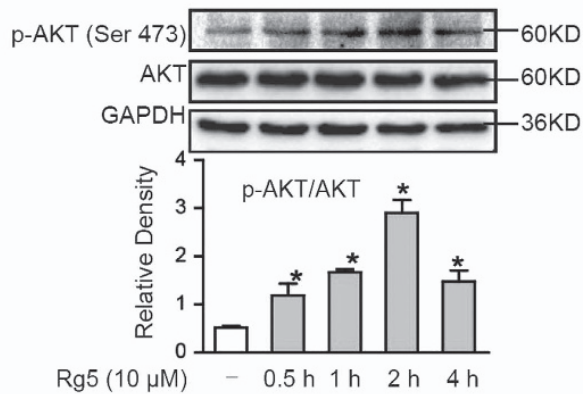

$\operatorname{Rg} 5(10 \mu \mathrm{M})$

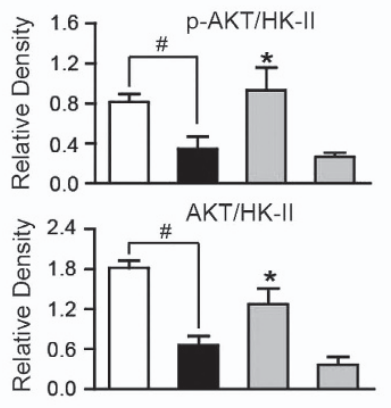

f

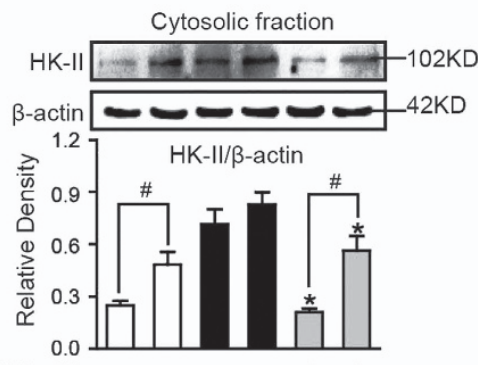

$\operatorname{Rg} 5(10 \mu \mathrm{M})$

PA $(100 \mu \mathrm{M})$

Control siRNA

AKT2 SIRNA
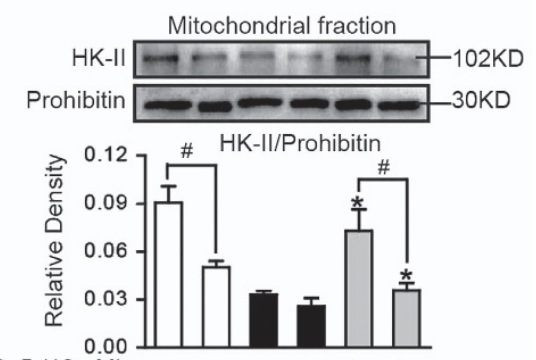

$\operatorname{Rg} 5(10 \mu \mathrm{M})$

$\mathrm{PA}(100 \mu \mathrm{M})$

Control siRNA

AKT2 siRNA

Figure 3 Rg5 promoted Akt association with HK-II in mitochondria. (a) Molecular docking analysis of the interaction between Rg5 and Akt. (b) Akt phosphorylation (S473) in neonatal rat ventricular myocytes (NRVMs) was determined by western blot. (c and d) NRVMs were stimulated with PA for $2 \mathrm{~h}$, and then PAS, Akt or p-Akt (S473) in HK-II were determined with immunoprecipitation and western blot. (e) NRVMs were stimulated with PA for $2 \mathrm{~h}$. Confocal image of HK-II and p-Akt (S473) colocalization in mitochondria were viewed with confocal scanning microscope (Blue: HK-Il; Green: p-Akt; Red: MitoTracker Red cMXRos. Scale bars: $10 \mu \mathrm{m}$ ); (f) H9c2 cells were transfected with Akt2 or control scrambled siRNA and then stimulated with palmitate (PA, $100 \mu \mathrm{M})$ for $2 \mathrm{~h}$. Mitochondrial and cytosolic HK-II expressions were determined using western blot. Data are expressed as mean \pm S.D. from three independent experiments. ${ }^{*} P<0.05$ versus control siRNA+PA treatment; ${ }^{\#} P<0.05$ versus the indicated treatment 

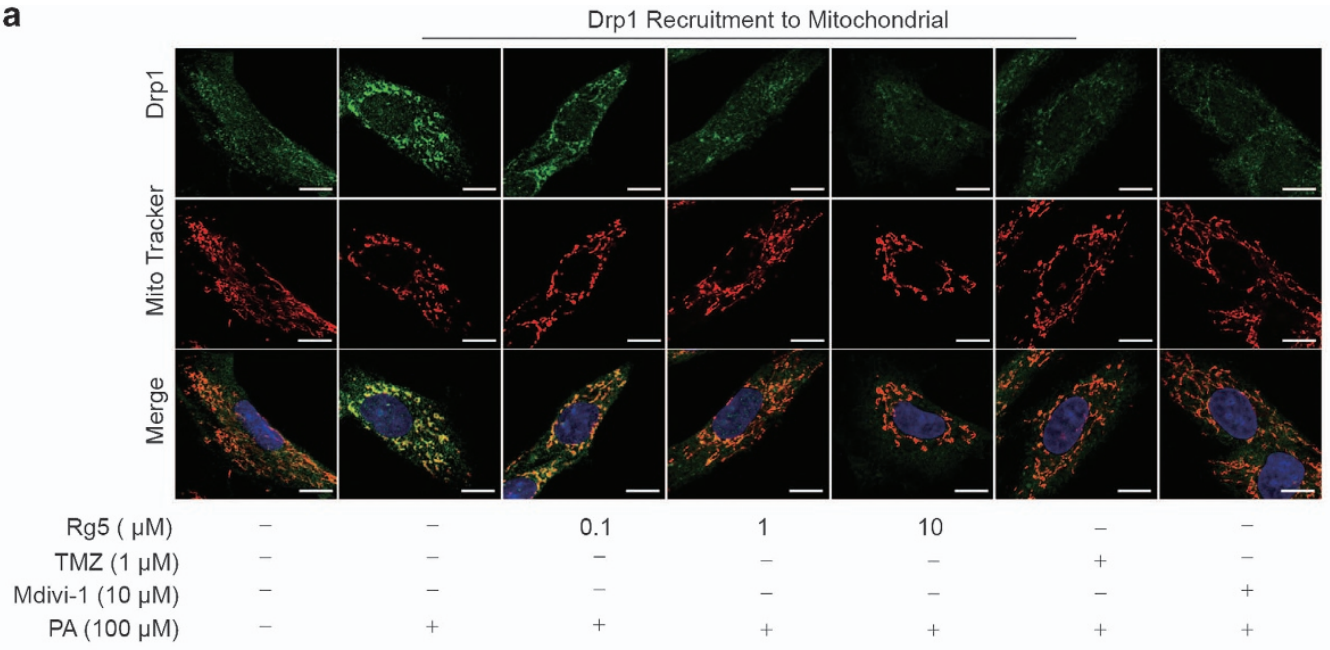

1

\section{0}

b

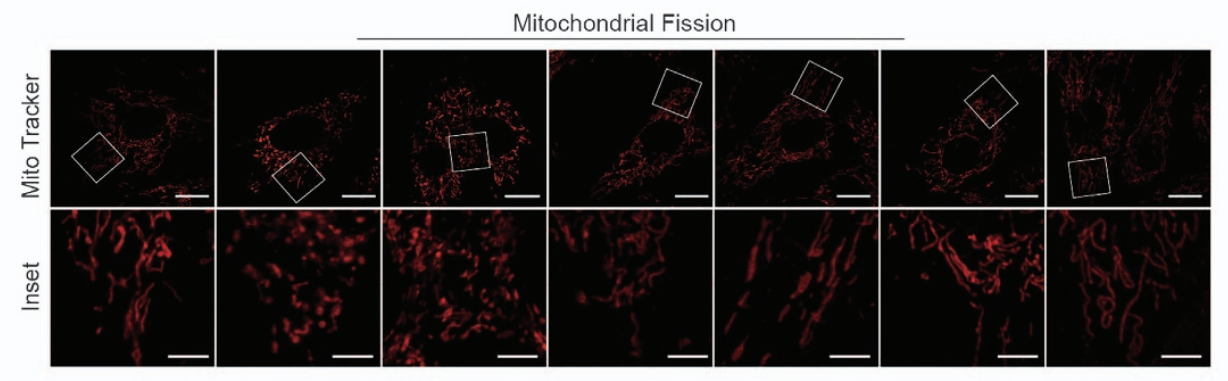

$\operatorname{Rg} 5(\mu \mathrm{M})$

$\operatorname{TMZ}(1 \mu \mathrm{M})$

Mdivi-1 $(10 \mu \mathrm{M})$

$\mathrm{PA}(100 \mu \mathrm{M})$
0.1

1

$-$
10

$-$ c

p-Drp1 (Ser637) $\square=-2$
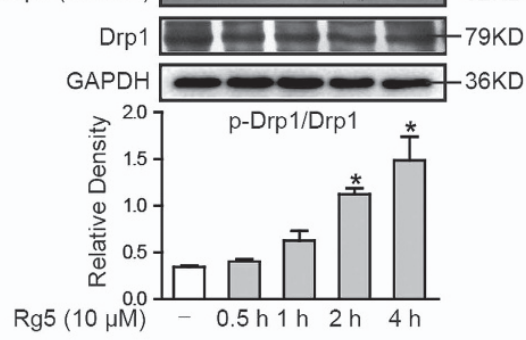

d

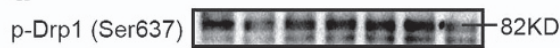

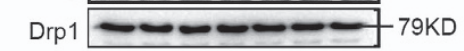
$\mathrm{GAPDH} \longrightarrow-\infty-\infty \mathrm{K}-\infty \mathrm{KD}$

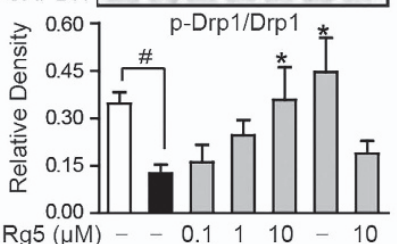
mito-TEMPO $(20 \mu \mathrm{M})$

Triciribine $(10 \mu \mathrm{M})$ $\mathrm{PA}(100 \mu \mathrm{M})$ KD

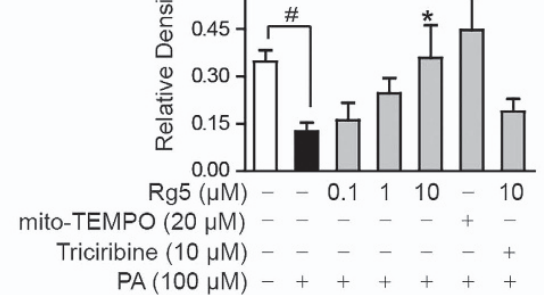

Figure 4 Rg5 inhibited Drp1 activation in cardiomyocytes. Neonatal rat ventricular myocytes (NRVMs) were stimulated with palmitate (PA, $100 \mu \mathrm{M})$ for $2 \mathrm{~h}$. (a) Confocal image of Drp1 in mitochondria (Green: Drp1; Red: MitoTracker Red cMXRos; Scale bars: $10 \mu \mathrm{m}$ ). (b) Mitochondrial fission was viewed by Mito Tracker Red CMXRos with confocal microscopy (Upper, scale bar, $10 \mu \mathrm{m}$; Lower, magnified images, bar, $5 \mu \mathrm{m}$ ). (c) The phosphorylation of Drp1 (S637) in NRVMs was determined by western blot; ${ }^{*} P<0.05$ versus the control. (d) Drp1 phosphorylation (S637) in NRVMs treated with PA for $2 \mathrm{~h} .{ }^{*} P<0.05$ versus PA treatment; ${ }^{*} P<0.05$ versus indicated treatment. (e) Drp1 phosphorylation (S637) in H9c2 cells transfected with Akt2 or control scrambled siRNA was determined by western blot. Data are expressed as mean \pm S.D. from three independent experiments. ${ }^{*} P<0.05$ versus control siRNA+PA treatment; ${ }^{\#} P<0.05$ versus indicated treatment

these actions contributed to protecting cardiomyocytes from I/R injury. We pretreated NRVMs with PA in the presence of $\mathrm{Rg} 5$ for $2 \mathrm{~h}$. After washing, cells were re-incubated under $1 \%$ $\mathrm{O}_{2}$ without glucose in the medium, followed by reoxygenation with glucose supply to mimic I/R insult. Oxygen/glucose depletion and reperfusion increased ROS production and calcium accumulation, whereas PA treatment augmented these responses (Figures $7 \mathrm{a}$ and b). Rg5 treatment suppressed ROS generation and reduced calcium accumulation (Figures 7a and b). As expected, Rg5 increased ATP content, prevented cytochrome $\mathrm{C}$ release from mitochondria and resultantly protected cells from apoptosis using flow cytometry analysis (Figures 7c-e). As a result, Rg5 promoted cardiomyocyte resistance to $\mathrm{l} / \mathrm{R}$ injury by protecting the 
a

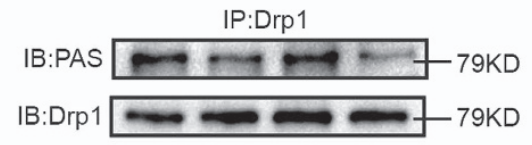

PAS/Drp1

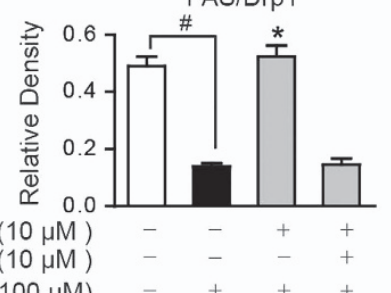

$\operatorname{Rg} 5(10 \mu \mathrm{M})$
PA $(100 \mu \mathrm{M})$

Triciribine $(10 \mu \mathrm{M})$
PA $(100 \mu \mathrm{M})$

b
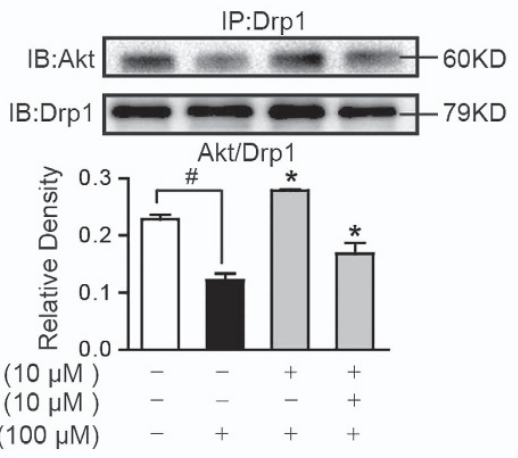

d

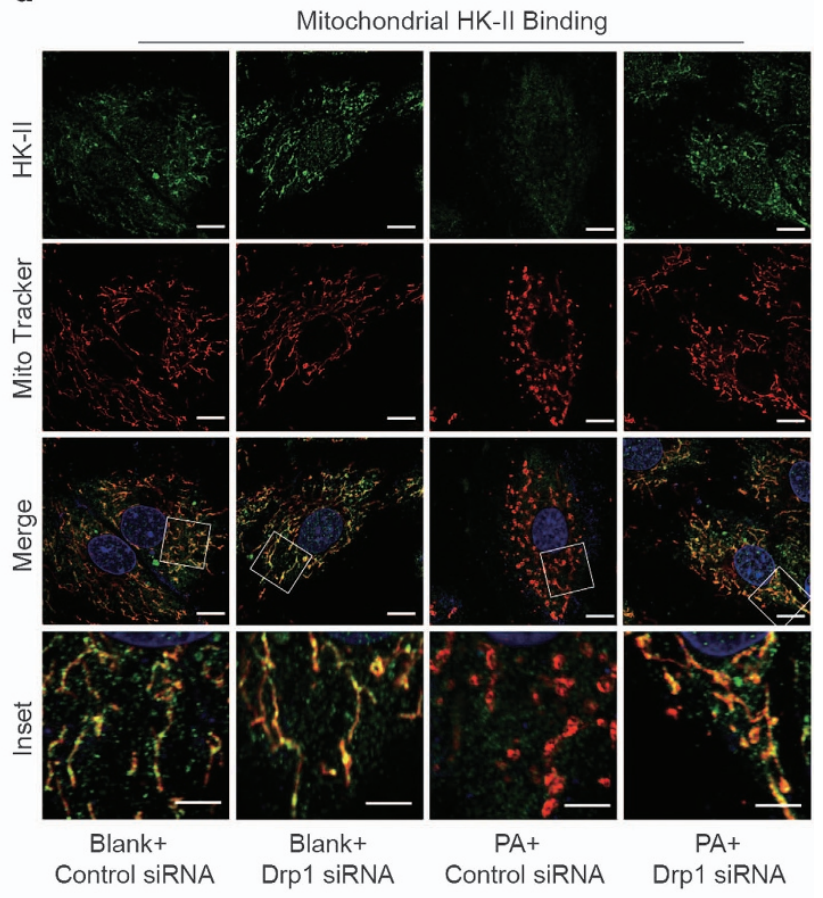

C

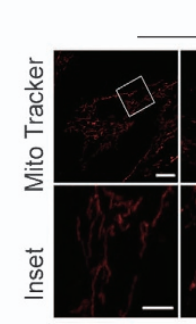

PA $(100 \mu \mathrm{M})$

Rg5 $(10 \mu \mathrm{M})$

Control siRNA

AKT2 siRNA
Mitochondrial Fission

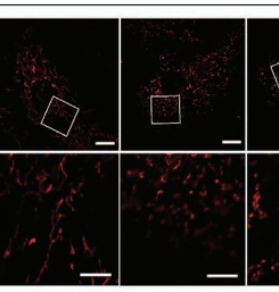

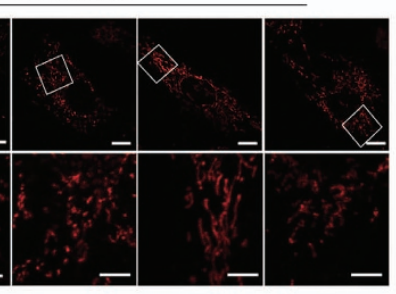

e

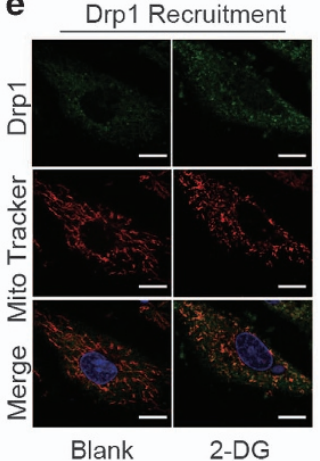

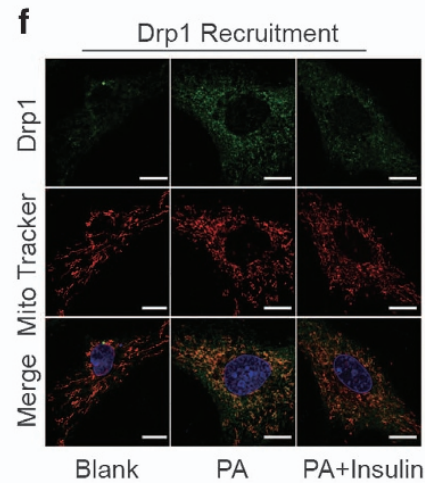

Figure 5 Regulation of Drp1 and HK-II in mitochondria. Neonatal rat ventricular myocytes (NRVMs) were stimulated with palmitate (PA,100 $\mu \mathrm{M})$ for $2 \mathrm{~h}$. (a and $\mathbf{b}$ ) Immunoprecipitation and western blot examination of PAS and Akt in precipitated Drp1 protein. ${ }^{*} P<0.05$ versus PA treatment; ${ }^{*} P<0.05$ versus indicated treatment. (c) Confocal scanning image of mitochondrial fission (Mito Tracker Red CMXRos). Upper, scale bar, $10 \mu \mathrm{m}$; Lower, magnified images, bar, $5 \mu \mathrm{m}$. (d) Confocal scanning image of mitochondrial HK-II expression in H9c2 cells transfected with Drp1 or control scrambled siRNA (Green: HK-II; Red: MitoTracker Red CMXRos; Upper, scale bar, $10 \mu \mathrm{m}$; Lower, magnified images, bar, $5 \mu \mathrm{m}$ ) (e and f) Confocal scanning image of mitochondrial Drp1 expression in NRVMs pretreated with 2-deoxy-D-glucose (2-DG, $5 \mathrm{mM})$ for $24 \mathrm{~h}$ or insulin $(0.1 \mu \mathrm{M})$ 30 min (Green: Drp1; Red: MitoTracker Red cMXRos; Scale bars: $10 \mu \mathrm{m}$ ). Data are expressed as mean \pm S.D. $(n=3)$. The results are confirmed by three independent experiments

structural and functional integrity of mitochondria. Together with above-mentioned results, our work demonstrated that Rg5 promoted HK-II binding to mitochondria and prevented Drp1 recruitment via regulation of Akt activation, thereby protecting cardiomyocytes against ischemic insult. The proposed pathway was shown in Figure $7 f$.

Rg5 protected mitochondrial integrity in isoproterenolinduced cardiac ischemia. To know the important role of mitochondrial integrity in the prevention of cardiac ischemic injury, we treated mice with isoproterenol to induce cardiac ischemia and observed the effect of Rg5 in vivo. Cardiac ischemia reduced Akt expression in cardiac mitochondria (Figure 8a), leading to the dissociation of HK-II from mitochondria (Figure 8b). Oral administration of Rg5 promoted Akt translocation to mitochondria and thus preserved mitochondrial HK-II (Figures 8a and b). Concordantly, Rg5 prevented Drp1 recruitment to mitochondria in the heart (Figure $8 \mathrm{c}$ ). As a result of the protection of mitochondrial integrity, Tunnel staining showed that Rg5 reduced cell apoptosis in ischemic heart, demonstrating its protective effect on cardiomyocyte survival (Figure 8d). Meanwhile, we observed that Rg5 reduced the elevated levels of blood free fatty acids in isoproterenol-treated 
a

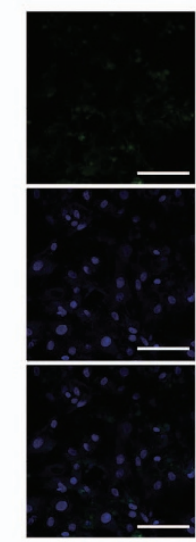

Blank
Intracelluar ROS Production

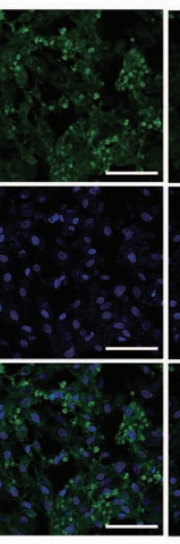

PA

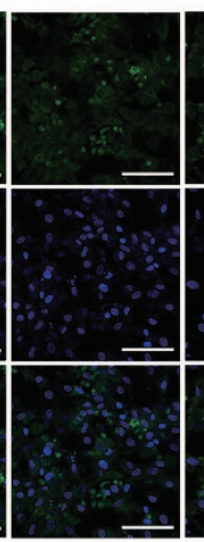

$\mathrm{PA}+$ $10 \mu \mathrm{M} \operatorname{Rg} 5$

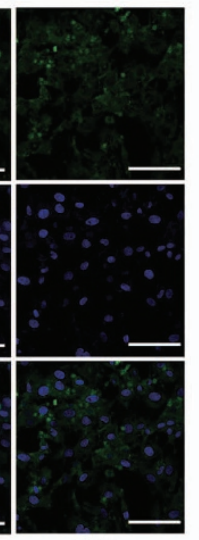

$\mathrm{PA}+$ $1 \mu \mathrm{M}$ TMZ b

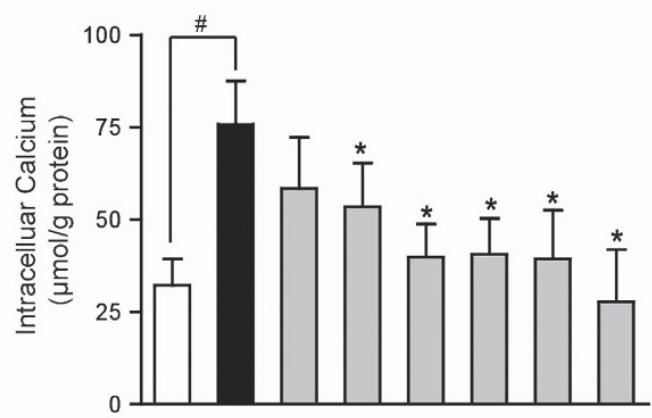

$\operatorname{Rg} 5(\mu \mathrm{M})$

$\operatorname{TMZ}(1 \mu \mathrm{M})$

Mdivi-1 $(10 \mu \mathrm{M})$ BAPTA-AM $(10 \mu \mathrm{M})$

$\mathrm{PA}(100 \mu \mathrm{M})$

c

Mitochondrial Transition Pore Opening

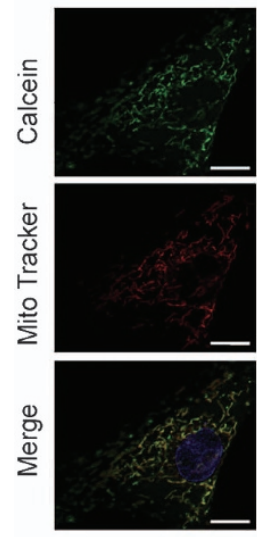

Blank
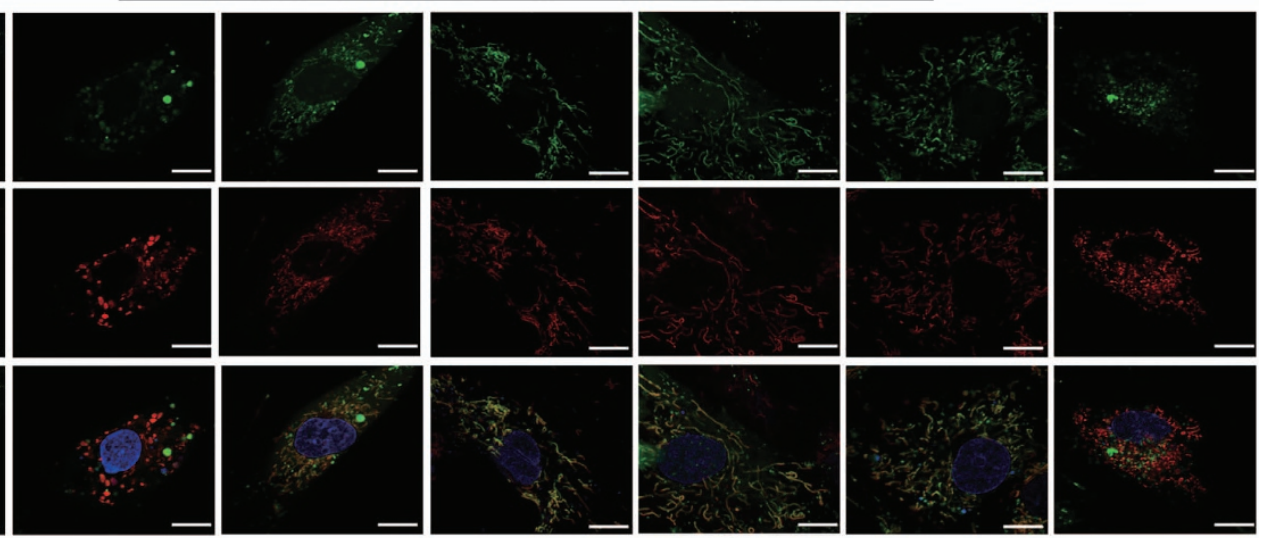

$\mathrm{PA}+$

$10 \mu \mathrm{M}$ Rg5

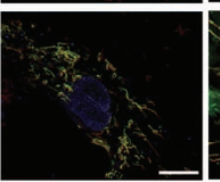

$\mathrm{PA}+$

$1 \mu \mathrm{MTMZ}$

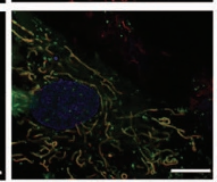

$\mathrm{PA}+$ $10 \mu \mathrm{M}$ Mdivi-1

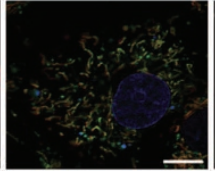

$\mathrm{PA}+$ $10 \mu \mathrm{M}$ CsA

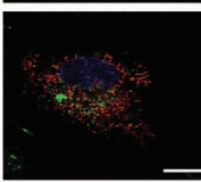

$1 \mu \mathrm{M}$ lonomycin

d

Mitochondrial Membrane Potential

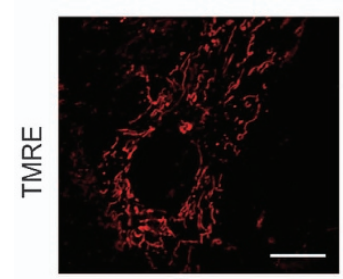

Blank

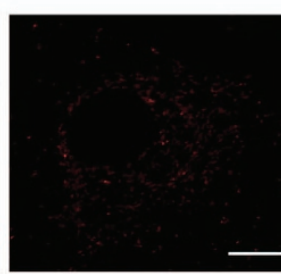

PA

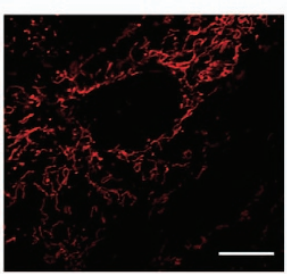

PA+

$10 \mu \mathrm{M} \operatorname{Rg} 5$

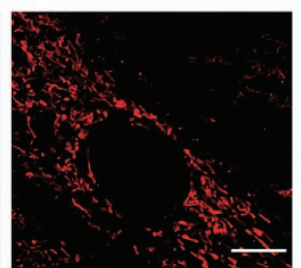

$\mathrm{PA}+$

$1 \mu \mathrm{M} \mathrm{TMZ}$

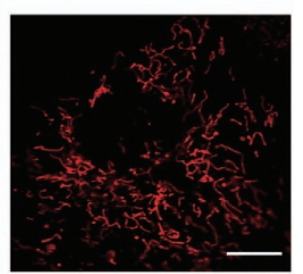

PA+

$10 \mu \mathrm{M}$ Mdivi-1

Figure 6 Rg5 ameliorated mitochondrial dysfunction in cardiomyocytes. Neonatal rat ventricular myocytes (NRVMs) were stimulated with palmitate (PA, $100 \mu \mathrm{M})$ for $2 \mathrm{~h}$. (a) Intracellular ROS production was viewed by dihydroethidium staining labeling with confocal microscopy. Scale bar, $100 \mu \mathrm{m}$; (b) Intracellular calcium concentration. ${ }^{*} P<0.05$ versus PA treatment; ${ }^{\#} P<0.05$ versus indicated treatment; Data are expressed as mean \pm S.D. $(n=4)$. (c) Calcein-AM combined with $\mathrm{CoCl}_{2}$ were used in viewing mitochondrial transition pore opening in cardiomyocytes. Scale bar, $10 \mu \mathrm{m}$. (d) Mitochondrial potential $(\Delta \psi \mathrm{m})$ was viewed by Tetramethylrhodamine ethyl ester perchlorate (TMRE) labeling with confocal microscopy. Scale bar, $10 \mu \mathrm{m}$. The results are from three independent experiments

mice (Supplementary Figure), a regulation potentially contributing to attenuating the impact of fatty acid on cardiac metabolism.

In parallel, we observed the impact of saturated acid challenge on mitochondrial integrity during $\mathrm{I} / \mathrm{R}$ insult in cardiomyocytes and found that pretreated PA reduced the content of mitochondrial HK-II and exaggerated mitochondrial fragments in response to hypoxia/reoxygenation insult (Figures $8 \mathrm{e}$ and $\mathrm{f}$ ). Rg5 treatment during PA challenge increased HK-II binding to mitochondria and prevented mitochondrial fission when cells were exposed to $1 \% \mathrm{O}_{2}$ and reoxygenation. These results further confirmed that maintenance of mitochondrial integrity was essential for resistance to ischemic injury. 
a

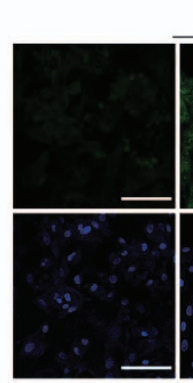

Blank
Intracelluar ROS Production

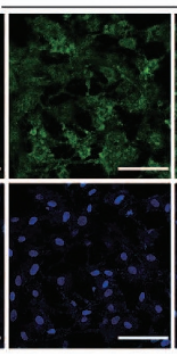

Only H/R

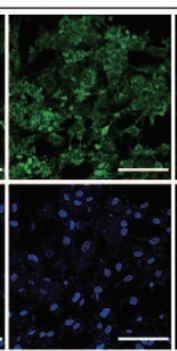

$\mathrm{PA}+\mathrm{H} / \mathrm{R}$

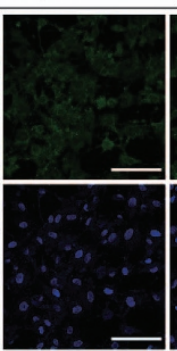

$\mathrm{PA}+\mathrm{H} / \mathrm{R}$

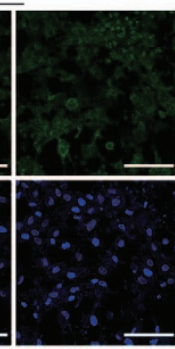

$\mathrm{PA}+\mathrm{H} / \mathrm{R}$ $+10 \mu \mathrm{M}$ Rg5 $+1 \mu \mathrm{M}$ TMZ

b

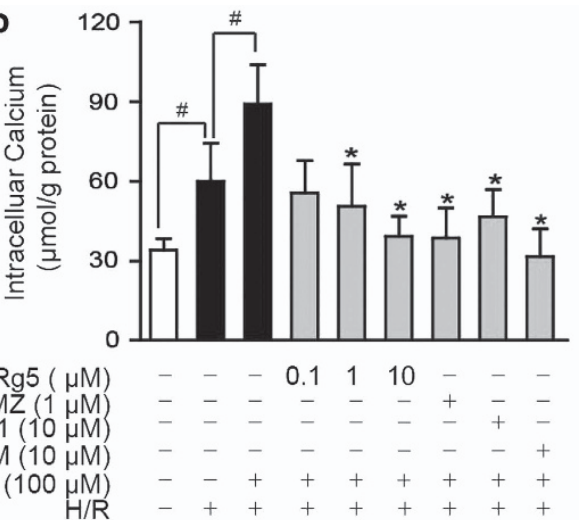

C

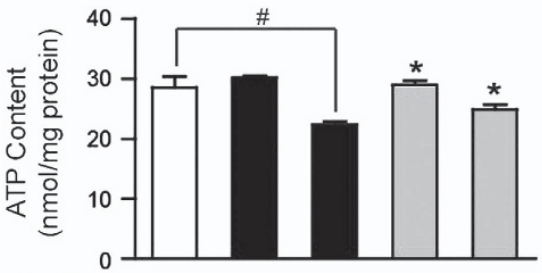

$\operatorname{Rg} 5(10 \mu \mathrm{M})$

$\mathrm{TMZ}(1 \mu \mathrm{M})$

PA $(100 \mu \mathrm{M})$

$H / R$ d

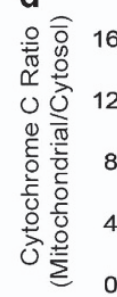

$\operatorname{Rg} 5(\mu \mathrm{M})$

$\operatorname{TMZ}(1 \mu \mathrm{M})$

$\mathrm{PA}(100 \mu \mathrm{M})$ e

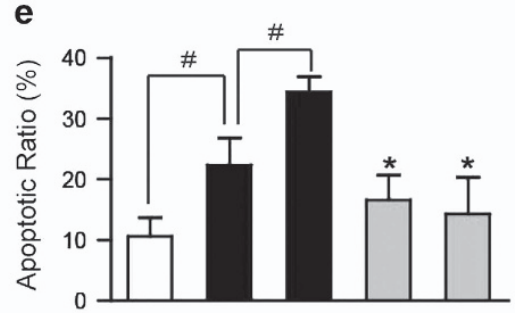

Rg5 $(10 \mu \mathrm{M})$

$\operatorname{TMZ}(1 \mu \mathrm{M})$

PA $(100 \mu \mathrm{M})$

H/R

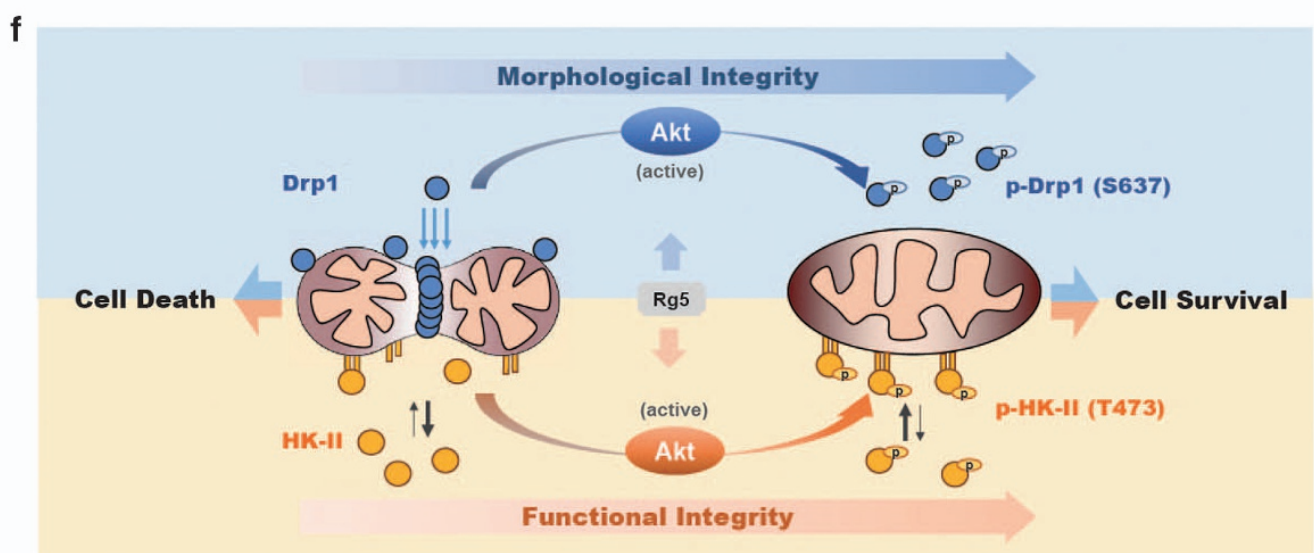

Figure 7 Rg5 increased cardiomyocyte resistance to I/R injury. Neonatal rat ventricular myocytes (NRVMs) were stimulated with palmitate (PA, $100 \mu \mathrm{M})$ for $2 \mathrm{~h}$ before $4 \mathrm{~h}$ hypoxia $\left(1 \% \mathrm{O}_{2}\right.$ ) and $1 \mathrm{~h}$ reoxygenation $(\mathrm{H} / \mathrm{R})$. (a) Intracellular ROS production in cardiomyocytes was viewed by dihydroethidium staining labeling with confocal microscopy. Scale bar, $100 \mu \mathrm{m}$. The results are confirmed by three independent experiments. Intracellular calcium concentration (b) and ATP content (c) were detected by commercial kits $(n=4)$. (d) The level of cytochrome $C$ in mitochondrial and cytosol fraction were measured by ELISA $(n=5)$. (e) The apoptosis assay was performed with AnnexinV/PI staining by flow cytometry analysis $(n=3)$. Data are expressed as mean \pm S.D. ${ }^{*} P<0.05$ versus $P A$ with $H / R$ treatment; ${ }^{*} P<0.05$ versus indicated treatment. (f) The proposed mechanism by which Akt activation prevented Drp1 recruitment and mitochondrial HK-II dissociation in cardiomyocyte

\section{Discussion}

HK-II binding to mitochondria protects cardiomyocyte survival by increasing energy efficiency. In contrast, Drp1 recruitment to mitochondria induces cell apoptosis through mitochondrial fission. In the current study, we found that HK-II and Drp1 competed for mitochondrial binding and their reciprocal control could be regulated by Akt. This finding suggests the potential role of Akt activation in cardioprotection.
Increased fatty-acid oxidation in mitochondria reduces carbohydrate oxidation by the uncoupling of glycolysis from pyruvate oxidation, and thus decreases cardiac efficiency in the use of energy. ${ }^{7} \mathrm{PDH}$ converts pyruvate into acetyl-CoA, thereby being key in linking glycolysis to pyruvate oxidation. In the present study, saturated acid challenge increased fattyacid oxidation in mitochondria accompanied by impaired PDH activity and lactate accumulation, indicating the inhibition of glucose oxidation by fatty-acid oxidation, a regulation also 
known as the Randle Cycle. ${ }^{2}$ As pyruvate oxidation is the major source for ATP production compared with glycolysis, the impaired glucose oxidation reduced heart energy efficiency.
It has been well documented that PDH is inhibited by NADH and acetyl-CoA produced from fatty-acid oxidation. ${ }^{28} \mathrm{Rg} 5$ reduced fatty-acid entry into mitochondria by downregulation
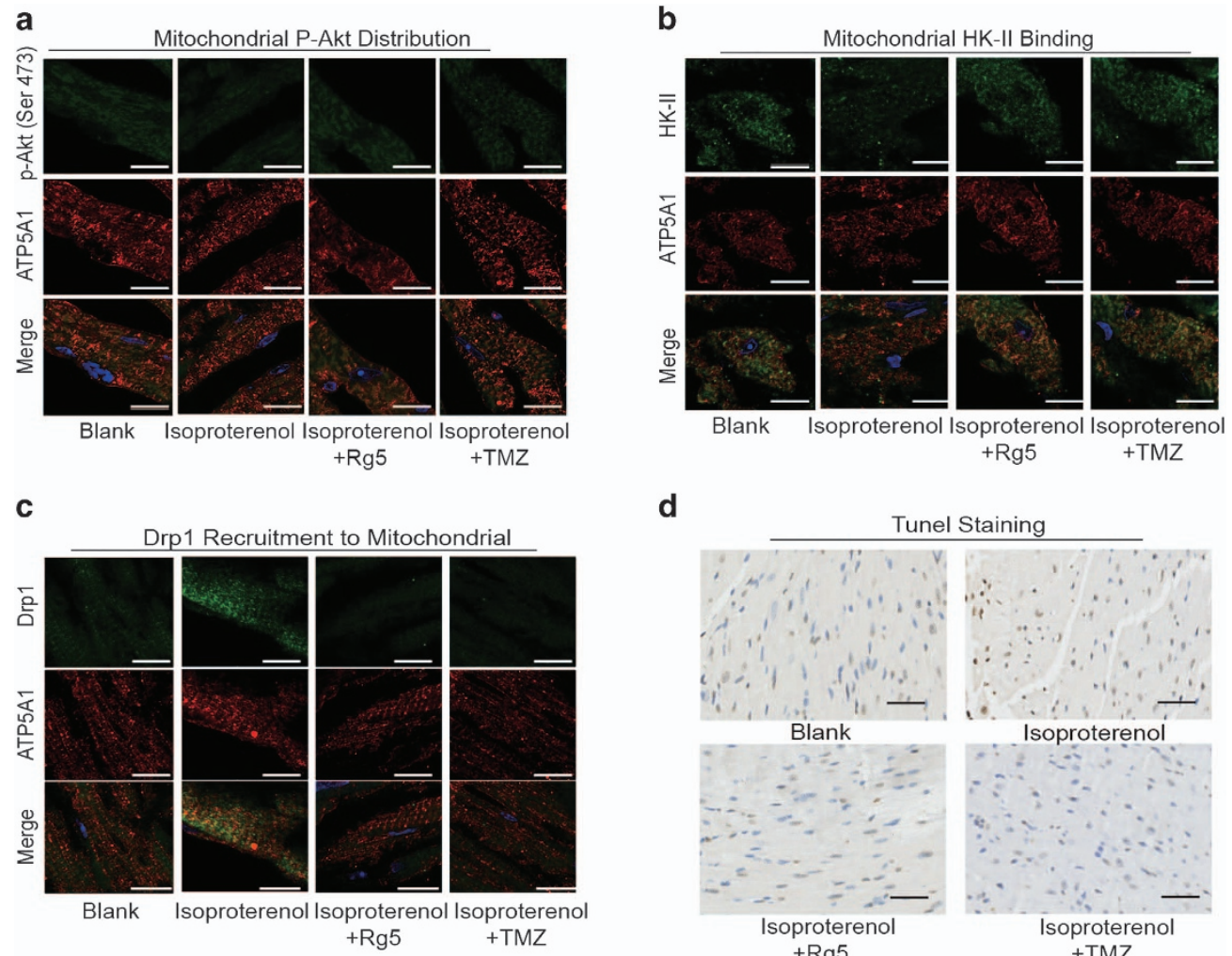

d

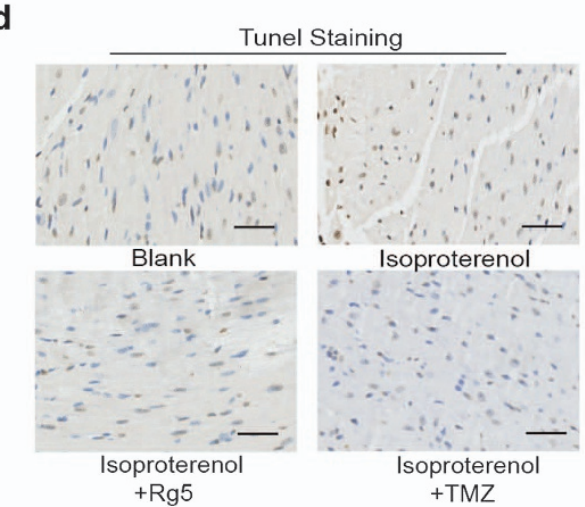

e

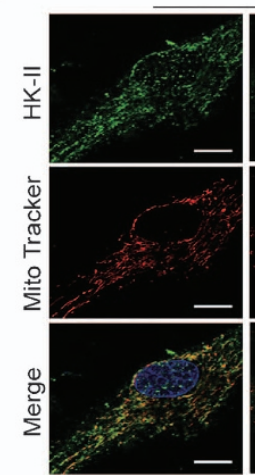

Blank

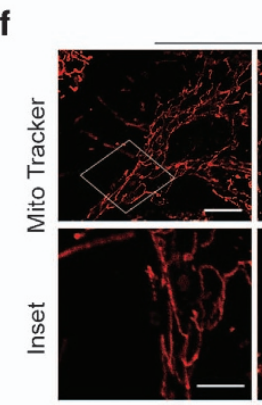

Blank

$+\mathrm{TMZ}$

Mitochondrial HK-II Binding
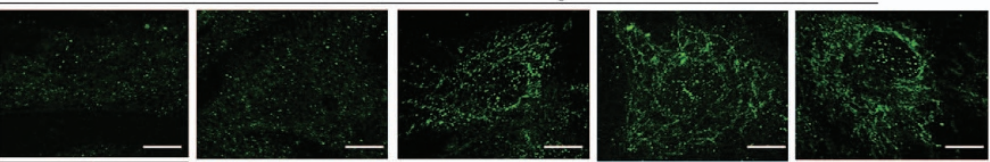

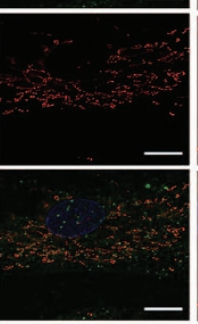

Only H/R

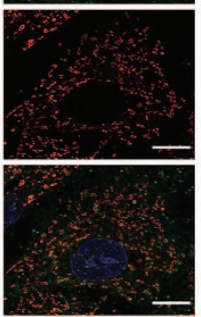

$\mathrm{PA}+\mathrm{H} / \mathrm{R}$

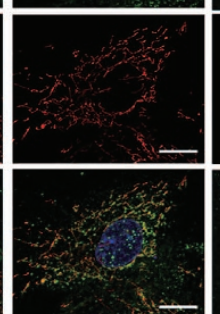

$\mathrm{PA}+\mathrm{H} / \mathrm{R}$

$+10 \mu \mathrm{M}$ Rg5

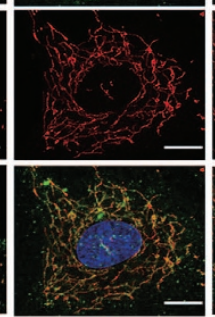

$\mathrm{PA}+\mathrm{H} / \mathrm{R}$ $+1 \mu \mathrm{M}$ TMZ

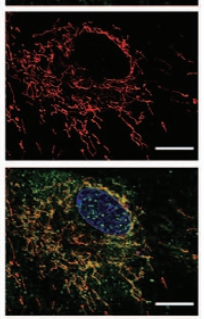

$\mathrm{PA}+\mathrm{H} / \mathrm{R}$ $+10 \mu \mathrm{M}$ Mdivi-1

Mitochondrial Fission

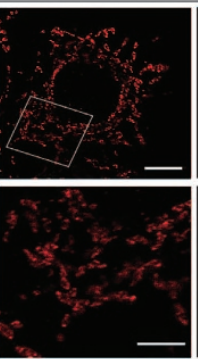

Only H/R

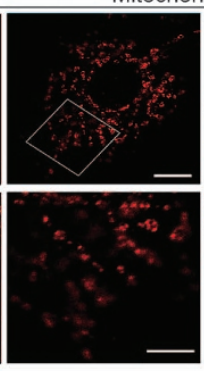

$\mathrm{PA}+\mathrm{H} / \mathrm{R}$

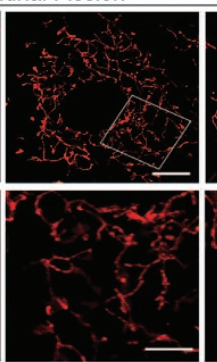

$\mathrm{PA}+\mathrm{H} / \mathrm{R}$ $+10 \mu \mathrm{M}$ Rg5

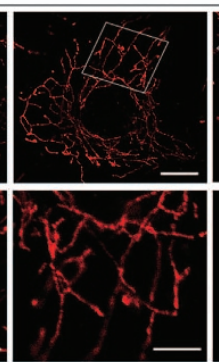

$\mathrm{PA}+\mathrm{H} / \mathrm{R}$ $+1 \mu \mathrm{M}$ TMZ

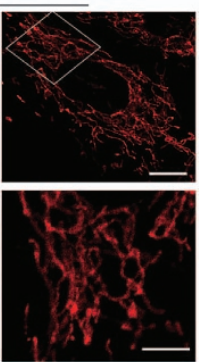

$\mathrm{PA}+\mathrm{H} / \mathrm{R}$ $+10 \mu \mathrm{M}$ Mdivi-1 
of CPT-1 and then decreased oxygen consumption in mitochondria, demonstrating its inhibitory effects on increased fatty-acid oxidation in mitochondria. Moreover, we showed that combating fatty-acid oxidation improved PDH activity, and thereby re-established the coupling of glycolysis and glucose oxidation, indicating the important role of PDH activity in the control of intracellular redox homeostasis.

It is generally considered that HK-II detachment from mitochondria mainly occurred during cardiac ischemia. ${ }^{29}$ Herein we showed that increased fatty-acid oxidation weakened HK-II binding to mitochondria owing to increased redox state, causing the enzyme to translocate to the cytoplasm. Rg5 inhibited fatty-acid oxidation in mitochondria and reduced lactate accumulation by restoring PDH activity, and thereby prevented HK-II dissociation from mitochondria. These findings indicate that the alternation of mitochondrial oxidation is an important cause for HK-II detachment from mitochondria.

Tumor cells show increased Akt activity which facilitates tumor survival through upregulation of HK expression. In tumor cells, HK-II expression and binding to mitochondria are regulated by $A k t / m T O R$ pathway. ${ }^{10,20}$ Therefore, the specific property in tumor survival raises the possibility that pharmacological activation of Akt might protect cardiomyocyte survival by increasing mitochondrial HK-Il binding. Consistent with this, it is documented that Akt activation prevents cardiomyocyte apoptosis induced by multiple pathological insults including ischemia reperfusion. ${ }^{30,31}$ Rg5 activated Akt in cardiomyocytes and induced Akt consensus PAS induction in HK-II protein, suggesting the possibility that Rg5 regulates mitochondrial HK-II through Akt activation. Akt traverses the cell interior with regulated localization, and consistent with this, we found that Rg5 treatment promoted Akt translocation to mitochondria and together with HK-II increased mitochondrial binding (Figure 3e). Akt inhibitor triciribine and knockdown of Akt blocked Rg5 action in the protection of mitochondrial HK-II, further confirming that Rg5 increased HK-Il binding to mitochondria via regulation of Akt activation. These results were consistent with a previous study showing that Akt increases mitochondrial HK-II association by phosphorylation of HK-II. ${ }^{22}$

The location of Akt in subcellular compartments is a key determinant of its biological effects. In addition to the regulation of $\mathrm{HK}-\mathrm{II}$, we found that $\mathrm{Rg} 5$ treatment induced PAS induction and increased activated Akt expression in Drp1 protein, indicating another molecular target regulated by Akt in mitochondria (Figures $5 a$ and b). In response to activation, the cytoplasmic Drp1 translocates to the mitochondrial surface for the fission reaction and induces cell death through mitochondria-dependent pathway in special tissues. ${ }^{32}$ Drp1 activation is regulated by modulating phosphorylation but Ser 637 phosphorylation is proposed to inactivate Drp1. ${ }^{18,19}$ Our work showed that Rg5 inhibited Drp1 activation and prevented mitochondrial fission by enhancing phosphorylation of Drp1 at Ser 637 in a manner dependent on Akt. Because mitochondrial integrity is essential for HK-II binding to mitochondria, it was convincible that Rg5 protected mitochondrial integrity by inhibition of mitochondrial fission, and thereby provided another way to increase mitochondrial HK-II. Drp1 inhibitor Mdivi-1 and gene knockdown of Drp1 protected mitochondrial HK-II, further supporting the conclusion.

Cytosolic HK-II and Drp1 translocate to mitochondria, demonstrating opposite effect on mitochondrial function. We found that inhibition of Drp1 activation increased mitochondrial HK-II. It was therefore worthwhile knowing if the binding of HKII to mitochondria prevented the recruitment of Drp1 to mitochondria. 2-DG is a modified glucose molecule that cannot undergo further glycolysis in cell. 2-DG mimics glucose 6-phosphate accumulation in cells and is usually used to induce HK-Il detachment from mitochondria, whereas insulin is considered to increase HK-II binding to mitochondria. In resting cells, Drp1 is inactive and mainly located in the cytoplasm. In the present study, when mitochondrial HK-II was reduced by 2-DG treatment, Drp1 expression in mitochondria increased. In contrast, insulin treatment inhibited PA-induced Drp1 recruitment to mitochondria. These results strongly suggested that Drp1 and HK-II controlled each other and competed for the binding to mitochondria. Consistent with the reciprocal control, Rg5 inhibited Drp1 recruitment to mitochondria and increased mitochondrial HK-Il via Akt activation, demonstrating the double targets to regulated by Akt in mitochondria.

Mitochondrial HK-II detachment and fission impaired mitochondrial integrity, leading to mitochondrial dysfunction in cardiomyocytes. Mitochondria are the main source of ROS production, though increased ROS amount could impair mitochondrial function through oxidative stress. Intracellular calcium accumulation is a resultant effect of altered ionic homeostasis. To re-establish ionic homeostasis, ATP is shifted away from contractile purpose to remove intracellular calcium, and the futile ATP consumption should be the main reason for the impairment of cardiac energy efficiency. Rg5 inhibited ROS production and reduced intracellular calcium accumulation, contributing to improving ionic homeostasis. Mitochondrial HK-II, together with VDAC and the ANT are essential for the maintenance of the permeability barrier of IMM. ${ }^{11} \mathrm{mPTP}$ is activated by $\mathrm{Ca}^{2+}$ together with phosphate and ROS, and the prolonged opening of $\mathrm{MPTP}$ can cause irreversible damage to the heart owing to inner membrane permeabilization, membrane potential dissipation, impaired PTP synthesis and the release of pro-apoptotic proteins. ${ }^{11}$ Consistently, accumulating evidence demonstrates that mitochondrial HK-II can confer protection against ischemic injury in the heart by limiting outer mitochondrial membrane permeabilization. ${ }^{33} \mathrm{PA}$ stimulation increased HK-II dissociation from mitochondria with the

Figure 8 Rg5 protected mitochondrial integrity in isoproterenol-induced ischemic heart of mice. (a-d) Mitochondrial p-Akt distribution (a), HK-II binding (b) and Drp1 recruitment $(\mathbf{c})$ in mitochondria were stained with immunofluorescence and viewed by confocal image $(n=4)$. Scale bar, $10 \mu \mathrm{m}$. (d) Heart paraffin section was stained with TUNEL $(n=3-6)$. Scale bar, $50 \mu \mathrm{m}$. Neonatal rat ventricular myocytes (NRVMs) were stimulated with palmitate (PA, $100 \mu \mathrm{M})$ for $2 \mathrm{~h}$ before $4 \mathrm{~h} \mathrm{hypoxia}\left(1 \% \mathrm{O}_{2}\right)$ and $1 \mathrm{~h}$ reoxygenation $(\mathrm{H} / \mathrm{R})$. Mitochondrial HK-II binding (e) and mitochondrial fission (f) were viewed by confocal image. Scale bar, $10 \mu \mathrm{m}$. The results are confirmed by three independent experiments 
opening of mPTP and the collapse of $\Delta \psi m$, setting cardiomyocytes at a stage susceptible to I/R damage. Indeed, we observed that PA-treated cardiomyocytes are more sensitive to mitochondrial dysfunction and apoptosis. As a result of the protection of mitochondrial function, Rg5 inhibited cytochrome $C$ release and reduced cell death during I/R insult. The preserved mitochondrial integrity and ATP production during I/R insult should be the important reasons for the resistance to cell death. Moreover, we developed cardiac ischemia in mice by injection of isoproterenol to confirm the cardioprotective effect of Rg5 in vivo. Isoproterenol-induced lipolysis increases fatty acid availability in the heart, well mimicking the impact of lipid challenge on cardiac metabolism. In the ischemic heart, Rg5 promoted Akt translocation to mitochondria, increased mitochondrial HK-II and prevented Drp1 recruitment, demonstrating its action in the protection of mitochondrial integrity. Similarly, we further confirmed the protective effects of Rg5 on mitochondrial integrity in PA-treated cardiomyocytes. As expected, we observed reduced cell apoptosis in ischemic heart. These results demonstrate that cardiac mitochondrial morphological and functional integrity is essential for resistance to ischemic injury.

In conclusion, the present study demonstrates that Drp1 and HK-II reciprocally control each other, competing for binding to mitochondria. Rg5 protects mitochondrial morphological and functional integrity by regulating HK-II and Drp1 translocation via Akt activation. Moreover, our work suggests that combating mitochondrial fatty-acid oxidation could reduce the susceptibility of the heart to ischemic injury in metabolic disorders.

\begin{abstract}
Materials and Methods
Materials. $\mathrm{Rg} 5$ (purity $=98 \%$ ) was a product of Jiangsu Yongjian Pharmaceutical Technology Co., Ltd. (Jiangsu, China). Tetramethylrhodamine ethyl ester perchlorate (TMRE), isoproterenol, 2-DG, trimetazidine and Mdivi-1 were from Sigma (St. Louis, MO, USA). Cyclosporin A and triciribine were purchased from Apex Bio (Houston, TX, USA). Mito-TEMPOL was purchased from Abcam (Cambridge, MA, USA). These agents were dissolved in dimethyl sulfoxide (DMSO) to obtain stock solutions and the final working concentration of DMSO was $<0.1 \%$ (v/v). PA (Sinopharm Chemical Reagent wq Co., Ltd. shanghai, China) was dissolved in ethanol to prepare $200 \mathrm{mM}$ stock solution and then was further diluted with medium containing 10\% FFA-free BSA at the ratio of 1:19 to obtain a concentration of $10 \mathrm{mM}$ before use. The following items were purchased from the cited commercial sources: anti-phospho-Drp1 (S637) (ab193216), anti-Drp1 (ab184247), anti-PDH E1 $\alpha$ (Ser 293) (ab177461), anti-CPT1A antibody (ab176320), Donkey Anti-Goat IgG H\&L (Alexa Fluor 647) (ab150131), Donkey Anti-Goat IgG H\&L (Alexa Fluor 405) (ab175664), anti-Prohibitin (ab75771), Abcam; anti-PDHA1 polyclonal antibody (BS7208), Goat Anti-Rabbit lgG $(\mathrm{H}+\mathrm{L})$ (Alexa Fluor 488) (BS13278), anti-GAPDH (AP0063), anti- $\beta$-Actin (AP0060), Bioworld Technology (St. Paul, MN, USA); anti-Hk-II (\#2867 S), anti-p-Akt (Ser 473) (\#4060), anti-Akt (\#4691), anti-Phospho-Akt Substrate (RXRXXS* $\left.T^{*}\right)$ (23C8D2), Cell Signaling Technology (Beverly, MA, USA).
\end{abstract}

Cell culture. NRVMs were isolated from 1-2-day-old Sprague-Dawley rat pups, digested with $0.08 \%$ collagenase and purified by differential adhesion method. Myocytes were maintained overnight in DMEM supplemented with $10 \%\left(\mathrm{v} / \mathrm{v}^{-1}\right)$ FBS at $37{ }^{\circ} \mathrm{C}$ in a humidified atmosphere of $5 \% \mathrm{CO}_{2}$. Cells were then serum starved for $2 \mathrm{~h}$ prior to intervention.

For PA treatment, cardiomyocytes were incubated with indicated agents for $0.5 \mathrm{~h}$, followed by PA $(100 \mu \mathrm{M})$ stimulation for $2 \mathrm{~h}$. To mimic ischemic injury in cell, the PAtreated cells were then washed with PBS and exposed to $1 \% \mathrm{O}_{2}$ with glucose deprivation for $4 \mathrm{~h}$. After that, the cells were re-incubated in a normal DMEM at $37^{\circ} \mathrm{C}$ in a $5 \% \mathrm{CO}_{2}$ humidified incubator for $1 \mathrm{~h}$.
Isoproterenol-induced myocardial ischemic injury in mice. Male ICR mice (18-22 g), supplied by the Laboratory Animal Center of Nanjing Qinglongshan, were housed in colony cages $12 \mathrm{~h} \mathrm{light/dark}$ cycles with free access to food and water. The animal care and experimental procedures were approved by Animal Ethics Committee of School of Chinese Materia Medica, China Pharmaceutical University. Rg5 $(50 \mathrm{mg} / \mathrm{kg})$ and TMZ $(20 \mathrm{mg} / \mathrm{kg})$ were administered orally $1 \mathrm{~h}$ before isoproterenol injection (120 mg/kg, subcutaneous) for 2 consecutive days. Animals in the sham groups received equivalent volume of $0.5 \%$ CMC-Na solution and saline in the same way. After $24 \mathrm{~h}$ of the last injection, blood sample was collected and heart tissue was obtained. For free fatty-acid assays, serum was separated by centrifuging at $3000 \mathrm{rpm}$ for $15 \mathrm{~min}$ and then detected with a commercial kit (Jiancheng Bioengineering Institute, Nanjing, China). For western blot analysis, left ventricle regions were quickly frozen in liquid nitrogen followed by storing at $-80^{\circ} \mathrm{C}$. For immunofluorescent assay and apoptosis assay, the whole heart was directly fixed in $4 \%$ paraformaldehyde or $15 \%$ sucrose.

Measurement of lactate, intracellular calcium, ATP content and NAD+/NADH ratio. NRVMs were treated with Rg5 or other agents at indicated concentrations, and then stimulated by PA or PA combined with hypoxia/ reoxygenation. After treatment, the supernatant was used for lactate assay using a commercial kit (Jiancheng Bioengineering Institute). Treated cells were collected, washed with PBS twice and lysed for measurements. Intracellular calcium concentration (Jiancheng Bioengineering Institute), ATP content (Beyotime Institute of Biotechnology, Shanghai, China) and NAD+/NADH ratio (Sigma) were detected with commercial kits respectively according to the manufacturer's protocol.

OCRs and ECRs measurements. OCRs were measured using the XFe96 Extracellular Flux Analyzer (Seahorse Bioscience, North Billerica, MA, USA). Cardiomyocytes were seeded (10 000 cells/well) and incubated overnight in DMEM supplemented with $10 \%\left(\mathrm{v} / \mathrm{v}^{-1}\right)$ FBS. One hour before the measurement, cells were incubated with $\mathrm{XF}$ medium at $37^{\circ} \mathrm{C}$ in a $\mathrm{CO}_{2}$-free incubator. The OCRs were detected under basal conditions and after the application of $1 \mu \mathrm{M}$ oligomycin, $0.5 \mu \mathrm{M}$ FCCP and $0.5 \mu \mathrm{M}$ rotenone $+0.5 \mu \mathrm{M}$ antimycin A (XF Cell Q17 Mito Stress Test Kit; Seahorse Bioscience). Each sample was analyzed four times.

Extracellular acidification rates (ECRs) were detected under basal conditions and after the application of $10 \mathrm{mM}$ glucose, $1 \mu \mathrm{M}$ oligomycin and $50 \mathrm{mM}$ 2-DG (XF Cell Q17 Glycolysis stress test kit; Seahorse Bioscience). Each sample was analyzed four times.

Molecular docking study. To evaluate the binding potential of Rg5 to Akt protein, in silico protein-ligand docking experiment was carried out by using the Autodock program (version 4.2) ${ }^{34}$ The structure of Akt was downloaded from the protein data bank (PDB ID: 1gzk), the crystal water molecules and other small molecules were removed. The Autodock program was then employed to generate an ensemble of docked conformations for Rg5 to Akt protein. We used the genetic algorithm for conformational search. To explore the conformational space of the Rg5 as completely as possible, we performed 100 individual genetic algorithm runs to generate 100 docked conformations. The size of the docking box was properly set to enclose the possible binding pocket. The protein structure was kept fixed during molecular docking.

Western blot analysis and immunoprecipitation. Cardiomyocytes were lysed in ice-cold RIPA buffer for $5 \mathrm{~min}$, incubated in an ice bath for $45 \mathrm{~min}$ and then cleared by centrifugation at $12000 \times \mathrm{g}$ for $20 \mathrm{~min}$ at $4^{\circ} \mathrm{C}$. Equal amounts of protein were electrophoresed on SDS-PAGE, transferred to a PVDF membrane and then blocked at room temperature for $2 \mathrm{~h}$. For immunoblotting, the primary antibodies were applied, respectively, at $4{ }^{\circ} \mathrm{C}$ overnight, followed by incubation with the secondary antibody at room temperature for $2 \mathrm{~h}$ or at $4{ }^{\circ} \mathrm{C}$ overnight. Western blot analyses were performed using anti-phospho-Drp1 (S637), anti-Drp1, anti-PDH E1 $\alpha$ (Ser 293), anti-CPT1A antibody, anti-Prohibitin; anti-PDHA1 polyclonal antibody, anti-GAPDH, anti- $\beta$-Actin, anti-Hexokinase-II, anti-p-Akt (Ser 473), antiAkt, anti-Phospho- Akt Substrate (RXRXXS* $\left./ T^{*}\right)$ specific antibodies. The values of band intensities were detected by enhanced chemiluminescence and quantized by Image-ProPlus 6.0 software.

For immunoprecipitation, NRVMs were treated with $\mathrm{Rg} 5(10 \mu \mathrm{M})$ plus or minus $\operatorname{TMZ}(10 \mu \mathrm{M})$ for $0.5 \mathrm{~h}$ before PA stimulation. After treatment cells were washed once with PBS and lysed for $15 \mathrm{~min}$ on ice. Cell lysates were then centrifuged at $12000 \times \mathrm{g}$ for $20 \mathrm{~min}$ and the soluble fraction was collected. Next, anti-Drp1 antibody or anti-HKII antibody was immunoprecipitated overnight at $4{ }^{\circ} \mathrm{C}$ and then with protein $\mathrm{A}+\mathrm{G}$ 
agarose beads (Beyotime Institute of Biotechnology) for another $2 \mathrm{~h}$. After that, the protein $A+G$ agarose beads were washed four times with the lysis buffer. The beads were then boiled in $1 \%$ SDS loading buffer for western blotting with the indicated antibodies.

Immunofluorescence. For the studies in heart from isoproterenol-induced mice, removed heart were fixed with $4 \%$ paraformaldehyde for $24 \mathrm{~h}$, dehydrated, embedded in Tissue-Tek O.C.T. Compound (Sakura Finetek, Torrance, CA, USA) and then cut in $8 \mu \mathrm{m}$ slices. The sections and NRVMs were washed with cold PBS, fixed $4 \%$ paraformaldehyde for 20 min. After that, NRVMs were permeabilized with $0.2 \%$ Triton X100 and incubated with 5\% BSA to block non-specific staining, and then incubated with specific primary antibodies staining (anti-Drp1, anti-HK2, antiphospho-Akt (Ser 473)) overnight at $4{ }^{\circ} \mathrm{C}$ in a humidified chamber. After several washings, the cells were incubated with Alexa Fluor 488-labeled goat anti-rabbit lgG $(\mathrm{H}+\mathrm{L})$ antibody, Donkey Anti-Goat IgG H\&L (Alexa Fluor 647), Donkey Anti-Goat IgG H\&L (Alexa Fluor 405) or Donkey Anti-Goat IgG H\&L (Alexa Fluor 488) for $1 \mathrm{~h}$ at $37^{\circ} \mathrm{C}$. They were then washed in PBS twice and incubated in DAPI for $15 \mathrm{~min}$ at $37^{\circ} \mathrm{C}$. The cells were mounted on a medium and visualized under a confocal scanning microscope.

The siRNA-mediated gene silencing. H9c2 cells (American Type Culture Collection, Manassas, VA, USA) were maintained in DMEM supplemented with $10 \%$ $\left(\mathrm{v} / \mathrm{v}^{-1}\right) \mathrm{FBS}$ at $37^{\circ} \mathrm{C}$ in a humidified atmosphere of $5 \% \mathrm{CO}_{2}$. To specifically suppress Drp1 and AKT2 expression, H9c2 cells were grown to $80 \%$ confluence and then transfected with siRNA duplexes specific for rat AKT2 (sc-108063, Santa Cruz Biotechnology, Santa Cruz, CA, USA), Drp1 (sc-43732, Santa Cruz Biotechnology) or control siRNA (sc-37007) by siRNA transfection reagent (sc-29528), respectively. After transfection, cells were cultured in medium for $48 \mathrm{~h}$. The cells were treated with indicated agents and PA $(100 \mu \mathrm{M})$ for $2 \mathrm{~h}$ and then collected and processed for immunofluorescence microscopy and western blot.

Cytosol/mitochondria fractionation. Cytosolic and mitochondrial fractions were prepared from NRVMs using Cell Mitochondria Isolation Kit (Beyotime Institute of Biotechnology) by the manufacturer's protocol. In brief, cells were collected by centrifugation at $650 \mathrm{~g}$ for $10 \mathrm{~min}$ at $4^{\circ} \mathrm{C}$, then the cells were washed twice with cold PBS and resuspended with Lysis buffer. After vortex and incubation on ice for $15 \mathrm{~min}$, the cells were homogenized for 25 stokes and the homogenates were centrifuged at $650 \mathrm{~g}$ for $10 \mathrm{~min}$ at $4{ }^{\circ} \mathrm{C}$. The supernatants were centrifuged at $11000 \times \mathrm{g}$ for $15 \mathrm{~min}$ at $4^{\circ} \mathrm{C}$. The resulting mitochondrial pellets were resuspended with mitochondrial lysis buffer. The supernatants were the cytosolic protein.

Mitochondrial fission, $\Delta \psi \mathrm{m}$ and ROS production assay. For mitochondrial fission assay, NRVMs were treated with Rg5, TMZ or Mdivi-1 at different concentrations, and then stimulated with PA or PA followed by hypoxia/ reoxygenation. After stimulation, the cells were washed with PBS and incubated with $200 \mathrm{nM}$ Mito Tracker Red CMXRos (Molecular Probes, Thermo Fisher Scientific, San Jose, CA, USA) for $30 \mathrm{~min}$ at $37^{\circ} \mathrm{C}$. The structure of mitochondria was viewed or measured using confocal microscopy (Zeiss LSM 700). For $\Delta \psi m$ assay, treated NRVMs were loaded with the potentiometric dye TMRE (final concentration of $500 \mathrm{nM}$ ) for $30 \mathrm{~min}$ at $37^{\circ} \mathrm{C}$. TMRE staining was examined by confocal microscopy (Zeiss LSM 700). For intracellular ROS detection, treated NRVMs were loaded with ROS-specific fluorescent probe dyedihydroethidium (Beyotime Institute of Biotechnology) and incubated for $0.5 \mathrm{~h}$ and DAPI (Sigma) for $15 \mathrm{~min}$ at $37^{\circ} \mathrm{C}$. After washing, cells were fixed in $4 \%$ paraformaldehyde for $5 \mathrm{~min}$ at $4{ }^{\circ} \mathrm{C}$ and viewed by confocal scanning microscopy (Zeiss LSM 700).

ELISA assay of cytochrome C. NRVMs were seeded in six-well plates, treated with Rg5 $(10 \mu \mathrm{M})$ or TMZ $(1 \mu \mathrm{M})$ and stimulated by PA under hypoxia/ reoxygenation. Cell cytosol and mitochondria fractions were collected and the concentration of cytochrome $C$ was assayed with commercial enzyme-linked immunosorbent assay (ELISA) kits (R\&D, USA).

Mitochondrial transition pore assay. NRVMs were treated with Rg5 $(10 \mu)$, TMZ $(1 \mu \mathrm{M})$, Mdivi-1 $(10 \mu \mathrm{M})$, cyclosporine $\mathrm{A}(10 \mu \mathrm{M})$ and stimulated by PA combined with hypoxia/reoxygenation. Inomycin $(1 \mu \mathrm{M})$ was added 30 min before detection. Mitochondrial transition pore opening was assayed with Image-IT LIVE Mitochondrial Transition Pore Assay Kit (Molecular Probes) by the manufacturer's protocol. Images were viewed by confocal scanning microscopy (Zeiss LSM 700).
Apoptosis analysis. NRVMs were treated with $\operatorname{Rg} 5(10 \mu \mathrm{M})$ and $\operatorname{TMZ}(1 \mu \mathrm{M})$ then stimulated with PA $(100 \mu \mathrm{M})$ under hypoxia/reoxygenation. After stimulation, cells were collected and detected using the AnnexinV-FITC Apoptosis Detection Kit (KeyGEN Biotech Co., Ltd. Nanjing, China). For apoptosis assay in NRVMs, cellular fluorescence was measured using flow cytometry analysis with a FACSCalibur Flow Cytometer (BD Biosciences, San Jose, CA, USA).

TUNEL assay. Cell death in the myocardium was assayed in heart paraffin section with an in situ Apoptosis Detection Kit based on the terminal deoxynucleotidyl transferase-mediated dUTP nick end-labeling (TUNEL) system (Roche Applied Science, Upper Bavaria, Germany).

Statistical analysis. The data are expressed as the mean \pm S.D. The significance of differences was analyzed by one-way ANOVA followed by the Bonferroni test. Differences were considered statistically significant at $P$-values of $\leqslant 0.05$.

\section{Conflict of Interest}

The authors declare no conflict of interest.

Acknowledgements. This study was supported in part by the Major Research Plan of the National Science Foundation of China (Grant No. 91639115), National Natural Science Foundation of China (81421005, 81573642), National Excellent Doctoral Dissertation of PR China (201278) and Jiangsu Province Science Fund for Distinguished Young Scholars (BK20130025). We thank the Cellular and Molecular Biology Center of China Pharmaceutical University for technical assistance. We thank Dr. Jin-Feng Liu for his technical support in molecular docking analysis. We also thank Dr. Raphael N Alolga for his assistance in proof-reading and correcting the manuscript.

1. Lopaschuk GD, Ussher JR, Folmes CD, Jaswal JS, Stanley WC. Myocardial fatty acid metabolism in health and disease. Physiol Rev 2010; 90: 207-258.

2. Randle PJ, Garland PB, Hales CN, Newsholme EA. The glucose fatty-acid cycle. Its role in insulin sensitivity and the metabolic disturbances of diabetes mellitus. Lancet 1963; 1: 785-789.

3. Herrero P, Peterson LR, McGill JB, Matthew S, Lesniak D, Dence C et al. Increased myocardial fatty acid metabolism in patients with type 1 diabetes mellitus. J Am Coll Cardiol 2006; 47: 598-604.

4. Carley AN, Severson DL. Fatty acid metabolism is enhanced in type 2 diabetic hearts. Biochim Biophys Acta 2005; 1734: 112-126.

5. Peterson LR, Herrero P, Schechtman KB, Racette SB, Waggoner AD, Kisrieva-Ware Z et al. Effect of obesity and insulin resistance on myocardial substrate metabolism and efficiency in young women. Circulation 2004; 109: 2191-2196.

6. Liu Q, Docherty JC, Rendell JC, Clanachan AS, Lopaschuk GD. High levels of fatty acids delay the recovery of intracellular $\mathrm{pH}$ and cardiac efficiency in post-ischemic hearts by inhibiting glucose oxidation. J Am Coll Cardiol 2002; 39: 718-725.

7. Fillmore N, Mori J, Lopaschuk GD. Mitochondrial fatty acid oxidation alterations in heart failure, ischaemic heart disease and diabetic cardiomyopathy. Br J Pharmacol 2014; 171 2080-2090.

8. Pedersen PL. Warburg, me and Hexokinase 2: multiple discoveries of key molecular events underlying one of cancers' most common phenotypes, the "Warburg Effect", i.e., elevated glycolysis in the presence of oxygen. J Bioenerg Biomembr 2007; 39: 211-222.

9. Wu R, Smeele KM, Wyatt E, Ichikawa Y, Eerbeek O, Sun L et al. Reduction in hexokinase II levels results in decreased cardiac function and altered remodeling after ischemia/ reperfusion injury. Circ Res 2011; 108: 60-69.

10. Robey RB, Hay N. Mitochondrial hexokinases, novel mediators of the antiapoptotic effects of growth factors and Akt. Oncogene 2006; 25: 4683-4696.

11. Kwong JQ, Molkentin JD. Physiological and pathological roles of the mitochondrial permeability transition pore in the heart. Cell Metab 2015; 21: 206-214.

12. Wang Z, Liu D, Varin A, Nicolas V, Courilleau D, Mateo P et al. A cardiac mitochondrial cAMP signaling pathway regulates calcium accumulation, permeability transition and cell death. Cell Death Dis 2016; 7: e2198.

13. Halestrap AP. What is the mitochondrial permeability transition pore? J Mol Cell Cardiol 2009; 46: 821-831.

14. Nederlof R, Eerbeek O, Hollmann MW, Southworth R, Zuurbier CJ. Targeting hexokinase II to mitochondria to modulate energy metabolism and reduce ischaemia-reperfusion injury in heart. Br J Pharmacol 2014; 171: 2067-2079.

15. Ong SB, Subrayan S, Lim SY, Yellon DM, Davidson SM, Hausenloy DJ. Inhibiting mitochondrial fission protects the heart against ischemia/reperfusion injury. Circulation 2010; 121: 2012-2022 
16. Barsoum MJ, Yuan H, Gerencser AA, Liot G, Kushnareva Y, Gräber S et al. Nitric oxideinduced mitochondrial fission is regulated by dynamin-related GTPases in neurons. EMBO J 2006; 25: 3900-3911.

17. Kanamaru Y, Sekine S, Ichijo H, Takeda K. The phosphorylation-dependent regulation of mitochondrial proteins in stress responses. J Signal Transduct 2012; 2012: 931215

18. Bhatt MP, Lim YC, Kim YM, Ha KS. C-peptide activates AMPK $\alpha$ and prevents ROSmediated mitochondrial fission and endothelial apoptosis in diabetes. Diabetes 2013; 62 : 3851-3862.

19. Li J, Wang Y, Wang Y, Wen X, Ma XN, Chen W et al. Pharmacological activation of AMPK prevents Drp1-mediated mitochondrial fission and alleviates endoplasmic reticulum stressassociated endothelial dysfunction. J Mol Cell Cardiol 2015; 86: 62-74.

20. Roberts DJ, Miyamoto S. Hexokinase II integrates energy metabolism and cellular protection: Akting on mitochondria and TORCing to autophagy. Cell Death Differ 2015; 22 248-257.

21. Miyamoto S, Murphy AN, Brown JH. Akt mediates mitochondrial protection in cardiomyocytes through phosphorylation of mitochondrial hexokinase-II. Cell Death Differ 2008; 15: 521-529.

22. Roberts DJ, Tan-Sah VP, Smith JM, Miyamoto S. Akt phosphorylates HK-II at Thr-473 and increases mitochondrial HK-II association to protect cardiomyocytes. J Biol Chem 2013; 288 : 23798-23806.

23. Shang W, Yang Y, Zhou L, Jiang B, Jin H, Chen M. Ginsenoside Rb1 stimulates glucose uptake through insulin-like signaling pathway in 3T3-L1 adipocytes. J Endocrinol 2008; 198 : 561-569.

24. Lee HM, Lee OH, Kim KJ, Lee BY. Ginsenoside Rg1 promotes glucose uptake through activated AMPK pathway in insulin-resistant muscle cells. Phytother Res 2012; 26: 1017-1022.

25. Shen L, Xiong Y, Wang DQ, Howles P, Basford JE, Wang J et al. Ginsenoside Rb1 reduces fatty liver by activating AMP-activated protein kinase in obese rats. $J$ Lipid Res 2013; 54 $1430-1438$.

26. Lee $\mathrm{CH}, \mathrm{Kim} \mathrm{JH}$. A review on the medicinal potentials of ginseng and ginsenosides on cardiovascular diseases. J Ginseng Res 2014; 38: 161-166.

27. Qi LW, Wang CZ, Yuan CS. Isolation and analysis of ginseng: advances and challenges. Nat Prod Rep 2011; 28: 467-495

28. Jaswal JS, Keung W, Wang W, Ussher JR, Lopaschuk GD. Targeting fatty acid and carbohydrate oxidation-a novel therapeutic intervention in the ischemic and failing heart. Biochim Biophys Acta 2011; 1813: 1333-1350.
29. Pasdois P, Parker JE, Halestrap AP. Extent of mitochondrial hexokinase II dissociation during ischemia correlates with mitochondrial cytochrome $c$ release, reactive oxygen species production, and infarct size on reperfusion. J Am Heart Assoc 2012; 2: e005645.

30. Fujio Y, Nguyen T, Wencker D, Kitsis RN, Walsh K. Akt promotes survival of cardiomyocytes in vitro and protects against ischemia-reperfusion injury in mouse heart. Circulation 2000; 101: 660-667.

31. Miao W, Luo Z, Kitsis RN, Walsh K. Intracoronary, adenovirus-mediated Akt gene transfer in heart limits infarct size following ischemia-reperfusion injury in vivo. J Mol Cell Cardiol 2000; 32: 2397-2402.

32. Sharp WW, Fang YH, Han M, Zhang HJ, Hong Z, Banathy A et al. Dynamin-related protein 1 (Drp1)-mediated diastolic dysfunction in myocardial ischemia-reperfusion injury: therapeutic benefits of Drp1 inhibition to reduce mitochondrial fission. FASEB J 2014; 28: 316-326.

33. Halestrap AP, Pereira GC, Pasdois P. The role of Hexokinase in cardioprotection mechanism and potential for translation. Br J Pharmacol 2015; 172: 2085-3000.

34. Morris GM, Goodsell DS, Halliday RS, Huey R, Hart WE, Belew RK et al. Automated docking using a Lamarckian genetic algorithm and an empirical binding free energy function. Comput Chem 1998; 19: 1639-1662.

(c) Cell Death and Disease is an open-access journal published by Nature Publishing Group. This work is licensed under a Creative Commons Attribution 4.0 International License. The images or other third party material in this article are included in the article's Creative Commons license, unless indicated otherwise in the credit line; if the material is not included under the Creative Commons license, users will need to obtain permission from the license holder to reproduce the material. To view a copy of this license, visit http://creativecommons.org/licenses/by/4.0/

(C) The Author(s) 2017

Supplementary Information accompanies this paper on Cell Death and Disease website (http://www.nature.com/cddis) 